Nafta-Gaz 2019, no. 10, pp. 598-612, DOI: 10.18668/NG.2019.10.02

\title{
Drilling muds with stable rheological parameters using modified natural polymers
}

\section{Płuczki wiertnicze o stabilnych parametrach reologicznych z wykorzystaniem modyfikacji polimerów naturalnych}

\author{
Grzegorz Zima \\ Oil and Gas Institute - National Research Institute
}

\begin{abstract}
This paper presents a study on the possible use of scleroglucan and guar gum mixture modified with aluminum ions as a part of a drilling mud. Currently, the most popular structure-directing agent for dispersed water-base drilling mud is the xanthan biopolymer (XCD). Scleroglucan is alternate structure-directing agent which can be used in a drilling mud, particularly in high temperature conditions. It has a completely nonionic character and creates a structure composed of polymer chains twisted in the form of a triple helix. Such build is characterized by high thermal resistance, multivalent metals cations resilience and salinity. Scleroglucan shows better capability in limiting water filtration than XCD especially in high temperatures. With that in mind its use is justified in the case of deep well. Improvement of rheologically-structural parameters of drilling muds can be achieved by using guar gum modified with use of trivalent ions, e.g. aluminum. This allows to reduce polymer concentration in drilling mud, resulting from a significant increase in viscosity due to the growth in molecular weight. Using scleroglucan in drilling mud composition combined with modified guar gum assures proper rheological parameters of drilling mud in low temperature as well as in high temperature. The combination of these two polymers enable the development of a drilling mud, which is characterized by the unique stability of its parameters in a wide temperature range. It is expected to reduce the expenditure on controlling drilling mud parameters during drilling due to combination of this type of agents in the drilling mud composition. In order to ensure an appropriate level of claystone hydration inhibition the addition of polyglycol was used in the composition of drilling muds, which in the proposed compositions additionally ensures appropriate technological parameters of drilling mud in borehole conditions. The paper proposes drilling mud compositions characterized by a special stability of rheological parameters in increased temperature. The most favorable properties of the drilling mud were obtained using a combination of xanthan, scleroglucan and guar gum modified with aluminum ions in its composition. By changing the type and concentration of individual polymers, we can design rheological parameters of drilling mud in the selected temperature range.
\end{abstract}

Key words: drilling mud, biopolymers, xanthan, guar gum, scleroglucan.

STRESZCZENIE: W artykule przedstawiono wyniki badań laboratoryjnych nad możliwością zastosowania w recepturach płuczek wiertniczych skleroglukanu w połączeniu z żywicą guarową modyfikowaną jonami glinu. Obecnie najpopularniejszym środkiem strukturotwórczym do płuczek wodnodyspersyjnych jest biopolimer ksantan (XCD). Alternatywnym środkiem strukturotwórczym, który może znaleźć zastosowanie w płuczkach wiertniczych, szczególnie w warunkach podwyższonej temperatury, jest skleroglukan. Posiada on całkowicie niejonowy charakter i tworzy strukturę złożoną z łańcuchów polimerowych skręconych w postaci potrójnej helisy. Taka budowa warunkuje jego dużą odporność termiczną oraz odporność na kationy metali wielowartościowych i zasolenie. Skleroglukan wykazuje również lepszą od XCD zdolność ograniczania filtracji, szczególnie w wysokich temperaturach, w związku z tym jego użycie jest uzasadnione w przypadku głębokich otworów. Poprawę parametrów reologiczno-strukturalnych płuczek można również uzyskać przez zastosowanie modyfikacji żywicy guarowej za pomocą jonów trójwartościowych, np. glinu. Umożliwia to zmniejszenie stężenia polimeru w płuczce, wynikającego ze znacznego wzrostu lepkości, spowodowanego wzrostem masy cząsteczkowej. Użycie skleroglukanu w składzie płuczki wiertniczej w połączeniu z modyfikowaną żywicą guarową zapewni odpowiednie parametry reologiczne płuczki zarówno w niskich, jak i wysokich temperaturach. Połączenie tych dwóch polimerów umożliwi opracowanie płuczki, która charakteryzuje się unikatową stabilnością parametrów w szerokim zakresie temperatury. W wyniku zastosowania połączenia tego rodzaju środków w składzie płuczki wiertniczej należy spodziewać się również zmniejszenia nakładów na kontrolowanie jej parametrów w czasie wiercenia. W celu zapewnienia odpowiedniego poziomu inhibicji hydratacji skał ilastych w płuczkach wiertniczych zastosowano dodatek poliglikolu, który w opracowanych składach dodatkowo zapewnia uzyskanie odpowiednich parametrów technologicznych płuczek wiertniczych w warunkach otworowych. W artykule zaproponowano składy płuczek wiertniczych charakteryzujących

Corresponding author: G. Zima, e-mail: grzegorz.zima@inig.pl

Article contributed to the Editor 10.01.2019. Approved for publication 05.09.2019 
się szczególną stabilnością parametrów reologicznych w podwyższonej temperaturze. Najkorzystniejsze właściwości płuczki uzyskano przy zastosowaniu w jej składzie połączenia ksantanu, skleroglukanu i guaru modyfikowanego jonami glinu. Zmieniając rodzaj i zawartości poszczególnych polimerów, możemy projektować parametry reologiczne płuczek w wybranym zakresie temperatur.

Słowa kluczowe: płuczka wiertnicza, biopolimery, ksantan, żywica guarowa, skleroglukan.

\section{Introduction}

In high temperature wellbore conditions, the values of rheological parameters decrease and the water filtration of the drilling mud increases. This is the result of physical changes occurring in the drilling mud under the influence of temperature or degradation of polymers "responsible" for providing proper rheological parameters to the drilling mud and limiting filtration. In the first case, it is a reversible process, while in the case of polymer degradation, continuous conditioning of the drilling mud is necessary. It is done by adding additional portions of polymer. The degradation of the polymer is particularly visible in the case of simultaneous occurrence of higher temperature and contamination with divalent ions $\left(\mathrm{Ca}^{2+}\right.$ and $\left.\mathrm{Mg}^{2+}\right)$. The contamination with divalent ions occurs mainly during the drilling through anhydrite $\left(\mathrm{CaSO}_{4}\right)$, gypsum $\left(\mathrm{CaSO}_{4} \cdot 2 \mathrm{H}_{2} \mathrm{O}\right)$, cement and carnallite $\left(\mathrm{KMgCl}_{3} \cdot 6 \mathrm{H}_{2} \mathrm{O}\right)$. For drilling in high temperature conditions, drilling muds containing polymers with increased thermal stability should be used, such as some synthetic polymers or modified natural polymers.

Currently, the most popular structuring agent for dispersed water-base drilling muds is the xanthan biopolymer (XCD). An alternative structuring agent that can be used in drilling muds compositions, especially at high temperature conditions is scleroglucan. It has a completely nonionic character and creates a structure composed of polymer chains twisted in the form of a triple helix. Such a construction determines high thermal resistance, multivalent metal cations resistance and salinity of the mud. Scleroglucan also shows better ability than xanthan for filtration control, especially at high temperatures. Improvement of rheologically-structural parameters of drilling mud can be achieved by using guar gum modified with addition of trivalent ions, e.g. aluminum. Using scleroglucan in drilling mud composition combined with modified guar gum assures proper rheological parameters of drilling mud in low temperature as well as in high temperature. The combination of these two polymers enables the development of a drilling mud, which is characterized by the unique stability of its parameters in a wide temperature range. In addition, the use of scleroglucan and guar gum biopolymer contributes to reducing the detrimental impact of the developed drilling mud on the permeability of reservoir formation and reducing the harmfulness on the natural environment due to their easy bio degradation. Furthermore, the rheological parameters of these drilling muds should be characterized by particular stability at high temperature, which will enable their use in a wider range of temperatures. The drilling muds contain xanthan as a structuring agent and polymers such as guar gum and scleroglucan are not used in Poland and are rarely used in the foreign market. The combined properties of both polymers has not been used in drilling mud compositions yet. It may prove to be an effective way to ensure stability of rheological parameters over a wide range of temperatures (Kulawardana et al., 1989; Wreath et al., 1990; Aston and Elliott, 1994; Fjelde and Stavland, 1995; Bielewicz and Bortel, 2000; El-Karsani et al., 2014a, 2014b).

\section{Biopolymers usage in drilling muds}

Xanthan gum (XCD) is currently the most popular biopolymer used as structuring agent to dispersed water-base drilling muds. As the bibliographical data shows (Cannell et al., 1988; Kalpakci et al., 1990; Riverq and Nolk, 1992; Gallino et al., 1996; Navarrete et al., 2000; Navarrete and Coffey, 2000; Lange and Keilhofer, 2004; Howard et al., 2015, Fortenberry et al., 2017) the alternative structuring agent that might be employed in drilling muds composition is scleroglucan bio synthesized with the participation of fungi of the genus Sklerotinum. Its chemical structure determines high thermal resistance and multivalent metal cations resistance and salinity. Maintaining proper values of rheological parameters of the drilling muds at high temperature is difficult due to the parameters being deteriorated which is caused by the increase of temperature and irreversible degradation of polymers. The use of scleroglucan in the composition of drilling muds contributes to the improvement of the rheological parameters of the drilling mud at high temperature and resistance to chemical contamination.

Natural polymers and their modifications are mainly used as the viscosity and water filtration control agents of drilling muds. However, they have lower thermal resistance and lower bacteria tolerance compared to the synthetic polymers. There is an increasing importance of polysaccharides in the drilling industry which are the products of enzymatic reactions carried out with the participation of bacteria and fungi referred to as biopolymers. These are plant resins extracted from plant material having specific rheological properties of their aqueous solutions. These solutions are characterized by high values of viscosity and gel strength at low concentrations. In addition, 
drilling fluids containing polymers of natural origin contribute to the prevention of damage to the near-wellbore zone (Dawson, 1991; Clark et al., 1993; de Kruijf et al., 1993; Rae, di Lullo, 1996; van Oort et al., 1997; Eriksen et al., 1997; Delshad et al., 2008; Seright et al., 2009; Steliga and Uliasz, 2012; Yerramilli et al., 2013; Elkarsani et al., 2014; Leonhardt et al., 2014; Li and Delshad, 2014; Ogezi et al., 2014 Brattekas et al., 2016; Behr and Rafiee, 2017).

Biopolymers and plant resins in a natural form often do not have sufficient thermal resistance and resilience to contamination with monovalent and divalent ions. Despite their use in low concentrations in the drilling mud, the cost of its preparation is high. Therefore, biopolymers and plant resins are often subjected to various chemical modifications. As a result of these transformations, the polymer takes on new properties and the range of its applications change accordingly. The improvement of gel strength and the possibility of reducing the concentration of natural polymer in the drilling mud (cost reduction) can be obtained by applying modifications with the use of appropriate metal ions. The possibility of reducing the polymer concentration in the drilling mud in this case is a result of significant increase in viscosity due to the molecular weight increase (Kramer et al., 1987; Harris, 1993; Himes et al., 1994; Queiroz and Santos, 2000; Lomba, 2002; Suresh Kumar et al., 2012; Dupas et al., 2013; Luyster et al., 2016; Zhu et al., 2017).

There are two features of the scleroglucan structure determining its special properties, the nonionic character and the twisted polymer chains into the triple helical structure. All authors of the publication (Kalpakci et al., 1990; Rivenq and Nolk, 1992; Fjelde and Stavland, 1995; Gallino et al., 1996; Navarreta et al., 2000) agree on the unique thermal resistance of scleroglucan but the results they present often differ. Their studies confirmed the thermal stability of scleroglucan at $90^{\circ} \mathrm{C}$ for 500 days and at $100^{\circ} \mathrm{C}$ for 60 days. Gallino et al. (1996) state that scleroglucan maintains its structure at a temperature higher than $120^{\circ} \mathrm{C}$, only at $130^{\circ} \mathrm{C}$ with $\mathrm{pH}$ of 7 , the viscosity decreases and the temperature does not depend on salinity. Research conducted by Kalpakci et al. (1990) confirmed the possibility of using scleroglucan at high temperatures. These authors determined that scleroglucan at $93^{\circ} \mathrm{C}$ does not change its properties even for several years, it can remain stable at temperatures of $105^{\circ} \mathrm{C}$, but for a correspondingly shorter period of time.

Drilling muds with the addition of scleroglucan were used during gas field exploration drilling in Southern Italy (Fjelde and Stavland, 1995). The used drilling mud contained 2-4 g/1 of biopolymer, 10-40 g/1 of potassium formate, 3-8 g/l of polymer for filtration control and barite for density control of $1,4-1,5 \mathrm{~g} / \mathrm{cm}^{3}$. While preparing the drilling mud, special attention was paid to mixing due to the nonionic nature of scleroglucan what resulting its slow hydration. During the preparation, the rheological parameters of the drilling mud were checked periodically. A significant influence of mixing intensity (circulation) on the hydration rate of the biopolymer was found. It was also observed that raising the $\mathrm{pH}$ above the value of 11.5 causes a significant deterioration the rheological parameters of the drilling mud, most probably caused by a change in the biopolymer conformation. Reducing the $\mathrm{pH}$ again results in a quick reconstruction of the drilling mud initial parameters. Drilling muds using scleroglucan and XCD polymer were compared for drilling wells located in the same area. The use of scleroglucan in place of XCD in drilling muds containing potassium formate allowed to reduce the time needed for works related to counteracting drilling bit bailing and the drilling mud conditioning by approximately $60 \%$. Industrial experiments confirmed scleroglucan resistance to salinity, contamination of dispersed solids and high temperature. The properties of the drilling mud were stable in the temperature range of $20-80^{\circ} \mathrm{C}$ and the $\mathrm{pH}$ in the range of 7-11. After heating the drilling mud during intensive circulation, rheological parameters were improved due to better biopolymer hydration. In the conditions of increased active particles of bentonite content, the drilling mud on the scleroglucan base showed better resistance to dispersed solids contamination than the drilling mud based on XCD polymer. Correlation studies were conducted between the content of active particles of bentonite (MBT) and Yield Point value (YP), in the case of drilling mud with scleroglucan the critical MBT value, i.e. at which a significant increase in Yield Point was observed was $65-70 \mathrm{~kg} / \mathrm{m}^{3}$, while in the case of drilling mud with XCD polymer, the critical MBT value was $50 \mathrm{~kg} / \mathrm{m}^{3}$.

Xanthan gum (XCD) is obtained by fermentation of a saccharide mixture using Xanthomas campestris bacteria. The viscosity of these solutions decreases when shear rate increases, with viscosity changes being immediate and reversible. This polymer is extremely stable $(\mathrm{pH}$ in the range of 2-10) and resistant to high chlorides concentrations $(\mathrm{NaCl}$ and $\mathrm{KCl})$ (Cannella et al., 1988; Wreath et al., 1990; Bielewicz and Bortel, 2000; Navarrete et al., 2000; Zima, 2001; Lange and Keilhofer, 2004; Yuan et al., 2010; Howard et al., 2015).

Guar gum is a plant resin extracted from the seed endosperm of guar bean seeds (Cyamopsis tetragonolobus). It forms stable aqueous solutions with high viscosity in the $\mathrm{pH}$ range of 4.0-10.5, with the fastest hydration process setting in at $\mathrm{pH} 8.0$. Guar plant is "compatible" with other plant resins and starch and as a result of a synergistic interaction with xanthan gum, viscosity increases is observed in mixed solutions (Dawson, 1991; Clark et al., 1993; de Kruijf et al., 1993; Shaari et al., 2005).

Attempts to apply chemical modifications of guar gum in the drilling mud technology are the result of actions to replace the biopolymer (XCD) with a cheaper agent with similar rheo- 
logical and structural properties. The Guar gum resin obtained from guar bean seeds after chemical modification, used in the drilling mud of a suitable composition, can be used as a structuring agent. The modification that meets the requirements of environmental protection and is characterized by high gel strength, ensuring effective removal and holding of cuttings is guar gum crosslinked with ions of nontoxic metals: aluminum and iron (III) (Kramer et al., 1987; Himes et al., 1994; Sydansk and Southwell, 2000; Zima, 2001; Zima and Błaż, 2004).

The use of guar gum has been limited to fracturing fluids and hydrogeological drilling fluids so far. The guar gum and hydroxypropyl guar crosslinked with boran ion, which is formed from the boric acid (III) molecule at high $\mathrm{pH}$ are used in fracturing fluids (Dawson, 1991; Clark et al., 1993; Eriksen et al., 1997; Taugbol et al., 2005; El-Karsani et al., 2014a, 2014b; Gaillard et al., 2015; Jouenne et al., 2015).

\section{Research on the selection of drilling mud composition containing scleroglucan and guar gum modified with aluminum ions}

The point of the research carried out at Oil and Gas Institute is to develop drilling muds with increased thermal resistance, by replacing the commonly used XCD biopolymer with scleroglucan or scleroglucan and guar gum modified with aluminum ions.
For this purpose, comparative tests of XCD polymer and scleroglucan in drilling muds at increased temperature and containing contaminants in the form of divalent metal ions were carried out. The method of guar gum modification and the composition of the drilling mud was selected on the basis of literature and tests previously conducted at Oil and Gas Institute (Zima and Błaż, 2004; Dawson, 1991; Clark et al., 1993). The drilling fluids used for the tests were prepared on the starch type protective colloids base with $0.3 \%$ additions one of the biopolymers.

After analyzing the properties of individual biopolymers, in order to obtain stable rheological parameters with the increase in temperature, a combination of several biopolymers in the composition of drilling muds was used. Drilling muds with the addition of biopolymers on the base of cellulose colloid had high filtration level, therefore for the further tests drilling muds prepared on the base of starch colloid were selected (Table 1). The best stability of rheological parameters and filtration under the influence of chemical contamination and temperature was found in drilling muds containing a combination of guar gum modified with aluminum ions, scleroglucan and XCD polymer (POS. 25, Table 1) and modified guar gum (POS. 13, Table 1). However, these drilling muds did not show a proper level of Miocene shale hydration inhibition (Fig. 1). Miocene shale recovery values after hydration $\left(P_{2}\right)$ were around $40 \%$. In order to improve the inhibition properties the addition of polyglycol was used in the composition of the drilling muds (Table 2).

Table 1. Influence of different additions of xanthan, scleroglucan and guar gum modified with aluminum ions on drilling muds properties Tabela 1. Wpływ różnych dodatków ksantanu, skleroglukanu i guaru modyfikowanego jonami glinu na właściwości płuczek wiertniczych

\begin{tabular}{|c|c|c|c|c|c|c|c|c|c|}
\hline \multirow{2}{*}{$\begin{array}{l}\text { No. } \\
\text { it. }\end{array}$} & \multirow{2}{*}{\multicolumn{2}{|c|}{$\begin{array}{c}\text { Composition } \\
{[\%]}\end{array}$}} & \multicolumn{2}{|c|}{$\begin{array}{l}\text { Viscosity } \\
{[\mathrm{mPa} \cdot \mathrm{s}]}\end{array}$} & \multirow{2}{*}{$\begin{array}{l}\text { Yield Point } \\
\text { [Pa] } \\
\text { YP }\end{array}$} & \multirow{2}{*}{$\begin{array}{c}\text { Filtration } \\
{\left[\mathrm{cm}^{3}\right]}\end{array}$} & \multicolumn{2}{|c|}{$\begin{array}{c}\text { Gel strength } \\
{[\mathrm{Pa}]}\end{array}$} & \multirow{2}{*}{ pH } \\
\hline & & & plastic & apparent & & & $\mathbf{I}$ & $\mathbf{U}$ & \\
\hline 1. & $\begin{array}{l}\text { Biostat } \\
\text { Starch agent } \\
\text { Xanthan } \\
\mathrm{KCl} \\
\text { Calcium carbonate }\end{array}$ & $\begin{array}{l}0.1 \\
3.0 \\
0.3 \\
7.0 \\
7.0\end{array}$ & 21 & 35.5 & 13.9 & 4.8 & 2.9 & 4.2 & 9.4 \\
\hline 2. & $\begin{array}{l}\text { Drilling mud } 1 \\
\text { Heating in an autoclave for } \\
16 \mathrm{~h} \text { temperature } 100^{\circ} \mathrm{C}\end{array}$ & & 17 & 29.5 & 12.0 & 3.2 & 1.6 & 2.4 & 8.6 \\
\hline 3. & $\begin{array}{l}\text { Drilling mud } 2 \\
+\mathrm{CaCl}_{2} \\
+\mathrm{NaOH}\end{array}$ & 1.0 & 16 & 26.5 & 10.3 & 4.4 & 0.9 & 1.8 & 9.1 \\
\hline 4. & $\begin{array}{l}\text { Drilling mud } 3 \\
+\mathrm{MgCl}_{2}\end{array}$ & 4.0 & 16 & 24.5 & 8.2 & 4.0 & 0.7 & 1.3 & 8.8 \\
\hline 5. & $\begin{array}{l}\text { Drilling mud } 4 \\
\text { heating in an autoclave for } \\
16 \mathrm{~h} \text { temperature } 100^{\circ} \mathrm{C} \\
+\mathrm{NaOH}\end{array}$ & & 14 & 22.0 & 4.4 & 4.8 & 0.8 & 1.4 & 8.9 \\
\hline 6. & $\begin{array}{l}\text { Drilling mud } 5 \\
+ \text { barite up to } 1,8 \mathrm{~g} / \mathrm{cm}^{3}\end{array}$ & & 71 & 98.5 & 26.3 & 4.3 & 4.6 & 7.7 & 8.8 \\
\hline
\end{tabular}




\section{NAFTA-GAZ}

ect. Table 1

\begin{tabular}{|c|c|c|c|c|c|c|c|c|c|}
\hline \multirow{2}{*}{$\begin{array}{l}\text { No. } \\
\text { it. }\end{array}$} & \multirow{2}{*}{\multicolumn{2}{|c|}{$\begin{array}{c}\text { Composition } \\
{[\%]}\end{array}$}} & \multicolumn{2}{|c|}{$\begin{array}{l}\text { Viscosity } \\
{[\mathrm{mPa} \cdot \mathrm{s}]}\end{array}$} & \multirow{2}{*}{$\begin{array}{l}\text { Yield Point } \\
\text { [Pa] } \\
\text { YP }\end{array}$} & \multirow{2}{*}{$\begin{array}{c}\text { Filtration } \\
{\left[\mathrm{cm}^{3}\right]}\end{array}$} & \multicolumn{2}{|c|}{$\begin{array}{c}\text { Gel strength } \\
{[\mathrm{Pa}]}\end{array}$} & \multirow{2}{*}{ pH } \\
\hline & & & plastic & apparent & & & $\mathbf{I}$ & U & \\
\hline 7. & $\begin{array}{l}\text { Biostat } \\
\text { Starch agent } \\
\text { Scleroglucan } \\
\mathrm{KCl} \\
\text { Calcium carbonate }\end{array}$ & $\begin{array}{l}0.1 \\
3.0 \\
0.3 \\
7.0 \\
7.0\end{array}$ & 18 & 25.0 & 6.9 & 4.0 & 1.4 & 2.3 & 9.7 \\
\hline 8. & $\begin{array}{l}\text { Drilling mud } 7 \\
\text { heating in an autoclave for } \\
16 \mathrm{~h} \text { temperatute } 100^{\circ} \mathrm{C}\end{array}$ & & 24 & 33.0 & 9.8 & 2.4 & 1.3 & 3.2 & 9.4 \\
\hline 9. & $\begin{array}{l}\text { Drilling mud } 8 \\
+\mathrm{CaCl}_{2} \\
+\mathrm{NaOH}\end{array}$ & 1.0 & 22 & 29.0 & 6.9 & 4.0 & 1.1 & 2.2 & 9.1 \\
\hline 10. & $\begin{array}{l}\text { Drilling mud } 9 \\
+\mathrm{MgCl}_{2}\end{array}$ & 4.0 & 21 & 29.0 & 7.9 & 2.8 & 1.3 & 2.4 & 9.4 \\
\hline 11. & $\begin{array}{l}\text { Drilling mud } 10 \\
\text { heating in an autoclave for } \\
16 \mathrm{~h} \text { temperature } 100^{\circ} \mathrm{C} \\
+\mathrm{NaOH}\end{array}$ & & 16 & 20.5 & 4.4 & 4.0 & 0.8 & 1.4 & 8.4 \\
\hline 12. & $\begin{array}{l}\text { Drilling mud } 11 \\
\text { + barite up to } 1.8 \mathrm{~g} / \mathrm{cm}^{3}\end{array}$ & & 69 & 95.5 & 25.4 & 4.3 & 4.5 & 7.8 & 8.2 \\
\hline 13. & $\begin{array}{l}\text { Biostat } \\
\text { Starch agent } \\
\text { Guar gum } \\
\mathrm{Al}_{2}\left(\mathrm{SO}_{4}\right)_{3} \cdot 18 \mathrm{H}_{2} \mathrm{O} \\
\mathrm{NaOH} \\
\mathrm{KCl} \\
\text { Calcium carbonate }\end{array}$ & $\begin{array}{c}0.1 \\
3.0 \\
0.3 \\
0.3 \\
0.18 \\
7.0 \\
7.0\end{array}$ & 20 & 41.0 & 20.1 & 3.0 & 3.9 & 4.9 & 9.5 \\
\hline 14. & $\begin{array}{l}\text { Drilling mud } 13 \\
\text { heating in an autoclave for } \\
16 \mathrm{~h} \text { temperature } 100^{\circ} \mathrm{C}\end{array}$ & & 28 & 49.5 & 20.6 & 3.2 & 3.6 & 3.7 & 9.1 \\
\hline 15. & $\begin{array}{l}\text { Drilling mud } 14 \\
+\mathrm{CaCl}_{2} \\
+\mathrm{NaOH}\end{array}$ & 1.0 & 26 & 46.0 & 19.2 & 4.0 & 2.4 & 2.7 & 8.6 \\
\hline 16. & $\begin{array}{l}\text { Drilling mud } 15 \\
+\mathrm{MgCl}_{2}\end{array}$ & 4.0 & 27 & 46.0 & 18.2 & 3.2 & 2.3 & 3.1 & 8.6 \\
\hline 17. & $\begin{array}{l}\text { Drilling mud } 16 \\
\text { heating in an autoclave for } \\
16 \mathrm{~h} \text { temperature } 100^{\circ} \mathrm{C} \\
+\mathrm{NaOH}\end{array}$ & & 23 & 31.0 & 6.4 & 4.0 & 0.7 & 1.4 & 8.9 \\
\hline 18. & $\begin{array}{l}\text { Drilling mud } 17 \\
+ \text { barite up to } 1.8 \mathrm{~g} / \mathrm{cm}^{3}\end{array}$ & & 72 & 98.5 & 26.4 & 4.0 & 4.5 & 8.8 & 8.7 \\
\hline 19. & $\begin{array}{l}\text { Biostat } \\
\text { Starch agent } \\
\text { Scleroglucan } \\
\text { Xanthan } \\
\mathrm{KCl} \\
\text { Calcium carbonate }\end{array}$ & $\begin{array}{c}0.1 \\
3.0 \\
0.15 \\
0.15 \\
7.0 \\
7.0\end{array}$ & 17 & 30.0 & 12.1 & 6.0 & 3.2 & 4.3 & 9.6 \\
\hline 20. & $\begin{array}{l}\text { Drilling mud } 19 \\
\text { heating in an autoclave for } \\
16 \mathrm{~h} \text { temperature } 100^{\circ} \mathrm{C}\end{array}$ & & 22 & 33.5 & 11.6 & 4.4 & 1.7 & 3.7 & 9.3 \\
\hline 21. & $\begin{array}{l}\text { Drilling mud } 20 \\
+\mathrm{CaCl}_{2} \\
+\mathrm{NaOH}\end{array}$ & 1.0 & 20 & 30.5 & 10.2 & 4.0 & 1.2 & 2.2 & 9.5 \\
\hline 22. & $\begin{array}{l}\text { Drilling mud } 21 \\
+\mathrm{MgCl}_{2}\end{array}$ & 4.0 & 21 & 30.5 & 9.3 & 6.4 & 1.3 & 2.6 & 9.3 \\
\hline
\end{tabular}


ect. Table 1

\begin{tabular}{|c|c|c|c|c|c|c|c|c|c|}
\hline \multirow{2}{*}{$\begin{array}{l}\text { No. } \\
\text { it. }\end{array}$} & \multirow{2}{*}{\multicolumn{2}{|c|}{$\begin{array}{c}\text { Composition } \\
{[\%]}\end{array}$}} & \multicolumn{2}{|c|}{$\begin{array}{l}\text { Viscosity } \\
{[\mathrm{mPa} \cdot \mathrm{s}]}\end{array}$} & \multirow{2}{*}{$\begin{array}{l}\text { Yield Point } \\
\text { [Pa] } \\
\text { YP }\end{array}$} & \multirow{2}{*}{$\begin{array}{c}\text { Filtration } \\
{\left[\mathrm{cm}^{3}\right]}\end{array}$} & \multicolumn{2}{|c|}{$\begin{array}{c}\text { Gel strength } \\
{[\mathrm{Pa}]}\end{array}$} & \multirow{2}{*}{ pH } \\
\hline & & & plastic & apparent & & & $\mathbf{I}$ & $\mathbf{U}$ & \\
\hline 23. & $\begin{array}{l}\text { Drilling mud } 22 \\
\text { heating in an autoclave for } \\
16 \mathrm{~h} \text { temperature } 100^{\circ} \mathrm{C} \\
+\mathrm{NaOH}\end{array}$ & & 12 & 17.5 & 5.4 & 4.8 & 0.7 & 1.3 & 8.4 \\
\hline 24. & $\begin{array}{l}\text { Drilling mud } 23 \\
+ \text { barite up to } 1.8 \mathrm{~g} / \mathrm{cm}^{3}\end{array}$ & & 69 & 96.5 & 26.9 & 4.8 & 4.0 & 6.8 & 8.7 \\
\hline 25. & $\begin{array}{l}\text { Biostat } \\
\text { Starch agent } \\
\text { Scleroglucan } \\
\text { Xanthan } \\
\text { Guar gum } \\
\mathrm{Al}_{2}\left(\mathrm{SO}_{4}\right)_{3} \cdot 18 \mathrm{H}_{2} \mathrm{O} \\
\mathrm{NaOH} \\
\mathrm{KCl} \\
\text { Calcium carbonate }\end{array}$ & $\begin{array}{c}0.1 \\
3.0 \\
0.1 \\
0.1 \\
0.1 \\
0.1 \\
0.06 \\
7.0 \\
7.0\end{array}$ & 21 & 26 & 14.8 & 4.0 & 3.3 & 6.1 & 9.6 \\
\hline 26. & $\begin{array}{l}\text { Drilling mud } 25 \\
\text { heating in an autoclave for } \\
16 \mathrm{~h} \text { temperature } 100^{\circ} \mathrm{C}\end{array}$ & & 26 & 40.5 & 14.3 & 4.0 & 1.8 & 3.5 & 9.3 \\
\hline 27. & $\begin{array}{l}\text { Drilling mud } 26 \\
+\mathrm{CaCl}_{2} \\
+\mathrm{NaOH}\end{array}$ & 1.0 & 23 & 34.5 & 11.3 & 4.0 & 1.3 & 2.6 & 9.2 \\
\hline 28. & $\begin{array}{l}\text { Drilling mud } 27 \\
+\mathrm{MgCl}_{2}\end{array}$ & 4.0 & 22 & 33 & 10.8 & 4.8 & 1.3 & 2.5 & 8.7 \\
\hline 29. & $\begin{array}{l}\text { Drilling mud } 28 \\
\text { heating in an autoclave for } \\
16 \mathrm{~h} \text { temperature } 100^{\circ} \mathrm{C}\end{array}$ & & 18 & 25 & 7.1 & 4.4 & 0.8 & 1.7 & 8.5 \\
\hline 30. & $\begin{array}{l}\text { Drilling mud } 29 \\
+ \text { barite up to } 1.8 \mathrm{~g} / \mathrm{cm}^{3}\end{array}$ & & 72 & 98.5 & 27.9 & 4.2 & 4.4 & 7.8 & 8.7 \\
\hline
\end{tabular}

Table 2. Different additives of xanthan, scleroglucan and guar gum modified with aluminum ions influence on drilling muds properties containing polyglycol

Tabela 2. Wpływ różnych dodatków ksantanu, skleroglukanu i guaru modyfikowanego jonami glinu na właściwości płuczek wiertniczych zawierających poliglikol

\begin{tabular}{|c|c|c|c|c|c|c|c|c|c|}
\hline \multirow{2}{*}{$\begin{array}{l}\text { No. } \\
\text { it. }\end{array}$} & \multirow{2}{*}{\multicolumn{2}{|c|}{$\begin{array}{c}\text { Composition } \\
{[\%]}\end{array}$}} & \multicolumn{2}{|c|}{$\begin{array}{l}\text { Viscosity } \\
{[\mathrm{mPa} \text { s] }}\end{array}$} & \multirow{2}{*}{$\begin{array}{c}\text { Yield Point } \\
\text { [Pa] } \\
\text { YP }\end{array}$} & \multirow{2}{*}{$\begin{array}{c}\text { Filtration } \\
{\left[\mathrm{cm}^{3}\right]}\end{array}$} & \multicolumn{2}{|c|}{$\begin{array}{c}\text { Gel strength } \\
{[\mathrm{Pa}]}\end{array}$} & \multirow{2}{*}{ pH } \\
\hline & & & $\begin{array}{l}\text { plastic } \\
\text { PV }\end{array}$ & $\begin{array}{c}\text { apparent } \\
\text { AV }\end{array}$ & & & I & II & \\
\hline 1 & $\begin{array}{l}\text { Biostat } \\
\text { Starch agent } \\
\text { Xanthan } \\
\mathrm{KCl} \\
\text { Calcium carbonate } \\
\text { Polyglycol }\end{array}$ & $\begin{array}{l}0.1 \\
3.0 \\
0.3 \\
7.0 \\
7.0 \\
4.0\end{array}$ & 18 & 31.5 & 13.0 & 4.8 & 4.0 & 5.3 & 9.4 \\
\hline 2 & $\begin{array}{l}\text { Drilling mud } 1 \\
\text { heating in an autoclave for } \\
16 \mathrm{~h} \text { temperature } 100^{\circ} \mathrm{C}\end{array}$ & & 15 & 27.5 & 12.0 & 2.8 & 2.3 & 3.9 & 8.8 \\
\hline 3 & $\begin{array}{l}\text { Drilling mud } 2 \\
+\mathrm{CaCl}_{2} \\
+\mathrm{NaOH}\end{array}$ & 1.0 & 16 & 26.0 & 9.6 & 2.4 & 1.7 & 2.9 & 9.2 \\
\hline 4 & $\begin{array}{l}\text { Drilling mud } 3 \\
+\mathrm{MgCl}_{2}\end{array}$ & 4.0 & 14 & 23.0 & 8.6 & 4.0 & 1.4 & 2.5 & 8.7 \\
\hline
\end{tabular}




\section{NAFTA-GAZ}

ect. Table 2

\begin{tabular}{|c|c|c|c|c|c|c|c|c|c|}
\hline \multirow{3}{*}{$\begin{array}{c}\text { No. } \\
\text { it. } \\
5\end{array}$} & \multirow{2}{*}{\multicolumn{2}{|c|}{$\begin{array}{c}\text { Composition } \\
{[\%]}\end{array}$}} & \multicolumn{2}{|c|}{$\begin{array}{l}\text { Viscosity } \\
\text { [mPa s] }\end{array}$} & \multirow{3}{*}{ 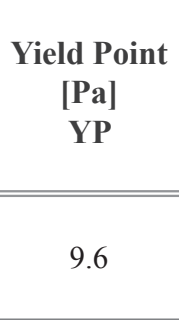 } & \multirow{3}{*}{$\begin{array}{c}\begin{array}{c}\text { Filtration } \\
{\left[\mathbf{c m}^{3}\right]}\end{array} \\
4.0\end{array}$} & \multicolumn{2}{|c|}{$\begin{array}{c}\text { Gel strength } \\
{[\mathrm{Pa}]}\end{array}$} & \multirow{3}{*}{$\begin{array}{l}\text { pH } \\
8.7\end{array}$} \\
\hline & & & $\begin{array}{l}\text { plastic } \\
\text { PV }\end{array}$ & $\begin{array}{c}\text { apparent } \\
\mathrm{AV}\end{array}$ & & & I & II & \\
\hline & $\begin{array}{l}\text { Drilling mud } 4 \\
\text { heating in an autoclave for } \\
16 \mathrm{~h} \text { temperature } 100^{\circ} \mathrm{C}+\mathrm{NaOH}\end{array}$ & & 14 & 24.0 & & & 1.6 & 1.8 & \\
\hline 6 & $\begin{array}{l}\text { Drilling mud } 5 \\
+ \text { barite up to } 1.8 \mathrm{~g} / \mathrm{cm}^{3}\end{array}$ & & 69 & 96.5 & 26.9 & 4.8 & 4.0 & 6.8 & 8.7 \\
\hline 7 & $\begin{array}{l}\text { Biostat } \\
\text { Starch agent } \\
\text { Scleroglucan } \\
\mathrm{KCl} \\
\text { Calcium carbonate } \\
\text { Polyglycol }\end{array}$ & $\begin{array}{l}0.1 \\
3.0 \\
0.3 \\
7.0 \\
7.0 \\
4.0\end{array}$ & 15 & 20.0 & 4.8 & 3.6 & 1.4 & 1.7 & 9.7 \\
\hline 8 & $\begin{array}{l}\text { Drilling mud } 7 \\
\text { heating in an autoclave for } 16 \mathrm{~h} \\
\text { temperature } 100^{\circ} \mathrm{C}\end{array}$ & & 20 & 30.0 & 9.6 & 3.6 & 2.4 & 5.3 & 9.4 \\
\hline 9 & $\begin{array}{l}\text { Drilling mud } 8 \\
+\mathrm{CaCl}_{2} \\
+\mathrm{NaOH}\end{array}$ & 1.0 & 17 & 23.5 & 6.4 & 3.6 & 1.1 & 3.1 & 9.7 \\
\hline 10 & $\begin{array}{l}\text { Drilling mud } 9 \\
+\mathrm{MgCl}_{2}\end{array}$ & 4.0 & 16 & 23.0 & 6.9 & 2.4 & 1.5 & 3.4 & 9.5 \\
\hline 11 & $\begin{array}{l}\text { Drilling mud } 10 \\
\text { heating in an autoclave for } 16 \mathrm{~h} \\
\text { temperature } 100^{\circ} \mathrm{C}\end{array}$ & & 12 & 18.0 & 5.9 & 4.4 & 1.5 & 3.2 & 8.8 \\
\hline 12 & $\begin{array}{l}\text { Drilling mud } 11 \\
+ \text { barite up to } 1.8 \mathrm{~g} / \mathrm{cm}^{3}\end{array}$ & & 69 & 96.5 & 26.9 & 4.8 & 4.0 & 6.8 & 8.7 \\
\hline 13 & $\begin{array}{l}\text { Biostat } \\
\text { Starch agent } \\
\text { Guar gum } \\
\mathrm{Al}_{2}\left(\mathrm{SO}_{4}\right)_{3} \cdot 18 \mathrm{H}_{2} \mathrm{O} \\
\mathrm{NaOH} \\
\mathrm{KCl} \\
\text { Calcium carbonate } \\
\text { Polyglycol }\end{array}$ & $\begin{array}{c}0.1 \\
3.0 \\
0.3 \\
0.3 \\
0.18 \\
7.0 \\
7.0 \\
4.0\end{array}$ & 16 & 31.0 & 14.4 & 4.8 & 2.2 & 2.7 & 9.5 \\
\hline 14 & $\begin{array}{l}\text { Drilling mud } 13 \\
\text { heating in an autoclave for } 16 \mathrm{~h} \\
\text { temperature } 100^{\circ} \mathrm{C}\end{array}$ & & 21 & 41.0 & 19.2 & 3.6 & 3.1 & 4.1 & 9.1 \\
\hline 15 & $\begin{array}{l}\text { Drilling mud } 14 \\
+\mathrm{CaCl}_{2} \\
+\mathrm{NaOH}\end{array}$ & 1.0 & 23 & 40.5 & 16.8 & 3.2 & 2.3 & 3.6 & 8.7 \\
\hline 16 & $\begin{array}{l}\text { Drilling mud } 15 \\
+\mathrm{MgCl}_{2}\end{array}$ & 4.0 & 22 & 38.5 & 15.8 & 4.0 & 2.1 & 2.7 & 8.7 \\
\hline 17 & $\begin{array}{l}\text { Drilling mud } 16 \\
\text { heating in an autoclave for } 16 \mathrm{~h} \\
\text { temperature } 100^{\circ} \mathrm{C}+\mathrm{NaOH}\end{array}$ & & 24 & 36.0 & 12.0 & 2.8 & 1.1 & 2.3 & 8.7 \\
\hline 18 & $\begin{array}{l}\text { Drilling mud } 17 \\
\text { + barite up to } 1.8 \mathrm{~g} / \mathrm{cm}^{3}\end{array}$ & & 69 & 96.5 & 26.9 & 4.8 & 4.0 & 6.8 & 8.7 \\
\hline 19 & $\begin{array}{l}\text { Biostat } \\
\text { Starch agent } \\
\text { Scleroglucan } \\
\text { Xanthan } \\
\mathrm{KCl} \\
\text { Calcium carbonate } \\
\text { Polyglycol }\end{array}$ & $\begin{array}{c}0.1 \\
3.0 \\
0.15 \\
0.15 \\
7.0 \\
7.0 \\
4.0\end{array}$ & 16 & 25.0 & 9.0 & 4.0 & 2.8 & 3.9 & 9.5 \\
\hline
\end{tabular}


ect. Table 2

\begin{tabular}{|c|c|c|c|c|c|c|c|c|c|}
\hline \multirow{2}{*}{$\begin{array}{l}\text { No. } \\
\text { it. }\end{array}$} & \multirow{2}{*}{\multicolumn{2}{|c|}{$\begin{array}{c}\text { Composition } \\
{[\%]}\end{array}$}} & \multicolumn{2}{|c|}{$\begin{array}{c}\text { Viscosity } \\
{[\mathrm{mPa} \text { s] }}\end{array}$} & \multirow{2}{*}{$\begin{array}{c}\text { Yield Point } \\
\text { [Pa] } \\
\text { YP }\end{array}$} & \multirow{2}{*}{$\begin{array}{c}\text { Filtration } \\
{\left[\mathrm{cm}^{3}\right]}\end{array}$} & \multicolumn{2}{|c|}{$\begin{array}{c}\text { Gel strength } \\
{[\mathrm{Pa}]}\end{array}$} & \multirow{2}{*}{ pH } \\
\hline & & & $\begin{array}{c}\text { plastic } \\
\text { PV }\end{array}$ & $\begin{array}{c}\text { apparent } \\
\text { AV }\end{array}$ & & & I & II & \\
\hline 20 & $\begin{array}{l}\text { Drilling mud } 19 \\
\text { heating in an autoclave for } 16 \mathrm{~h} \\
\text { temperature } 100^{\circ} \mathrm{C}\end{array}$ & & 21 & 31.0 & 10.9 & 3.2 & 2.4 & 4.8 & 9.3 \\
\hline 21 & $\begin{array}{l}\text { Drilling mud } 20 \\
+\mathrm{CaCl}_{2} \\
+\mathrm{NaOH}\end{array}$ & 1.0 & 17 & 27.0 & 9.9 & 4.0 & 1.9 & 3.6 & 9.4 \\
\hline 22 & $\begin{array}{l}\text { Drilling mud } 21 \\
+\mathrm{MgCl}_{2}\end{array}$ & 4.0 & 17 & 27.0 & 9.9 & 4.8 & 1.9 & 3.7 & 9.2 \\
\hline 23 & $\begin{array}{l}\text { Drilling mud } 22 \\
\text { heating in an autoclave for } \\
16 \mathrm{~h} \text { temperature } 100^{\circ} \mathrm{C}\end{array}$ & & 15 & 23.0 & 7.9 & 4.8 & 1.5 & 2.6 & 8.9 \\
\hline 24 & $\begin{array}{l}\text { Drilling mud } 23 \\
+ \text { barite up to } 1.8 \mathrm{~g} / \mathrm{cm}^{3}\end{array}$ & & 69 & 96.5 & 26.9 & 4.8 & 4.0 & 6.8 & 8.7 \\
\hline 25 & $\begin{array}{l}\text { Biostat } \\
\text { Starch agent } \\
\text { Scleroglucan } \\
\text { Xantan } \\
\text { Guar gum } \\
\mathrm{Al}_{2}\left(\mathrm{SO}_{4}\right)_{3} \cdot 18 \mathrm{H}_{2} \mathrm{O} \\
\mathrm{NaOH} \\
\mathrm{KCl} \\
\text { Calcium carbonate } \\
\text { Polyglycol }\end{array}$ & $\begin{array}{c}0.1 \\
3.0 \\
0.1 \\
0.1 \\
0.1 \\
0.1 \\
0.06 \\
7.0 \\
7.0 \\
4.0\end{array}$ & 17 & 28.5 & 11.4 & 3.6 & 2.9 & 5.2 & 9.6 \\
\hline 26 & $\begin{array}{l}\text { Drilling mud } 25 \\
\text { heating in an autoclave for } \\
16 \mathrm{~h} \text { temperature } 100^{\circ} \mathrm{C}\end{array}$ & & 25 & 39.0 & 13.9 & 2.8 & 2.8 & 4.9 & 9.3 \\
\hline 27 & $\begin{array}{l}\text { Drilling mud } 26 \\
+\mathrm{CaCl}_{2} \\
+\mathrm{NaOH}\end{array}$ & 1.0 & 20 & 32.0 & 11.9 & 4.4 & 1.9 & 4.3 & 9.0 \\
\hline 28 & $\begin{array}{l}\text { Drilling mud } 27 \\
+\mathrm{MgCl}_{2}\end{array}$ & 4.0 & 21 & 32.0 & 10.9 & 4.4 & 1.8 & 4.3 & 8.9 \\
\hline 29 & $\begin{array}{l}\text { Drilling mud } 28 \\
\text { heating in an autoclave for } 16 \mathrm{~h} \\
\text { temperature } 100^{\circ} \mathrm{C}\end{array}$ & & 17 & 25.5 & 8.4 & 4.8 & 1.3 & 2.8 & 8.7 \\
\hline 30 & $\begin{array}{l}\text { Drilling mud } 29 \\
+ \text { barite up to } 1.8 \mathrm{~g} / \mathrm{cm}^{3}\end{array}$ & & 69 & 96.5 & 26.9 & 4.8 & 4.0 & 6.8 & 8.7 \\
\hline
\end{tabular}

The polyglycol addition positively influenced the values of $P_{2}$, which in the tested drilling muds amounted to about 60\% (Fig. 2). Furthermore, after the addition of polyglycol an improvement in the stability of rheological parameters and a decrease in water filtration were observed. Drilling muds with compositions proposed in Table 1 and Table 2 were used in rheological parameters stability study in the temperature range of $20-100^{\circ} \mathrm{C}$ with the use of HPHT OFI Testing Equipment 77 viscometer.

The outcome from dispersion analysis of Eocene and Miocene Shale (Fig. 1 and 2) confirm the high degree of formations hydration inhibition for all tested drilling muds. Shale recovery both Eocene and Miocene from the drilling mud reach values close to $100 \%(92-100 \%)$, both in drilling muds with addition of polyglycol and without it. Drilling muds without polyglycol additives protect formation against water to a lesser extent, shale recovery from water amount to about $40 \%$ for the Miocene Shale. The addition of polyglycol increases the formation recovery from water $\left(P_{2}\right)$ to about $60 \%$. The obtained results do not allow to point out which polysaccharide inhibits hydration of shales the most, because they are comparable for individual drilling muds. Only the addition of polyglycol has an influence on $P_{2}$ value. 

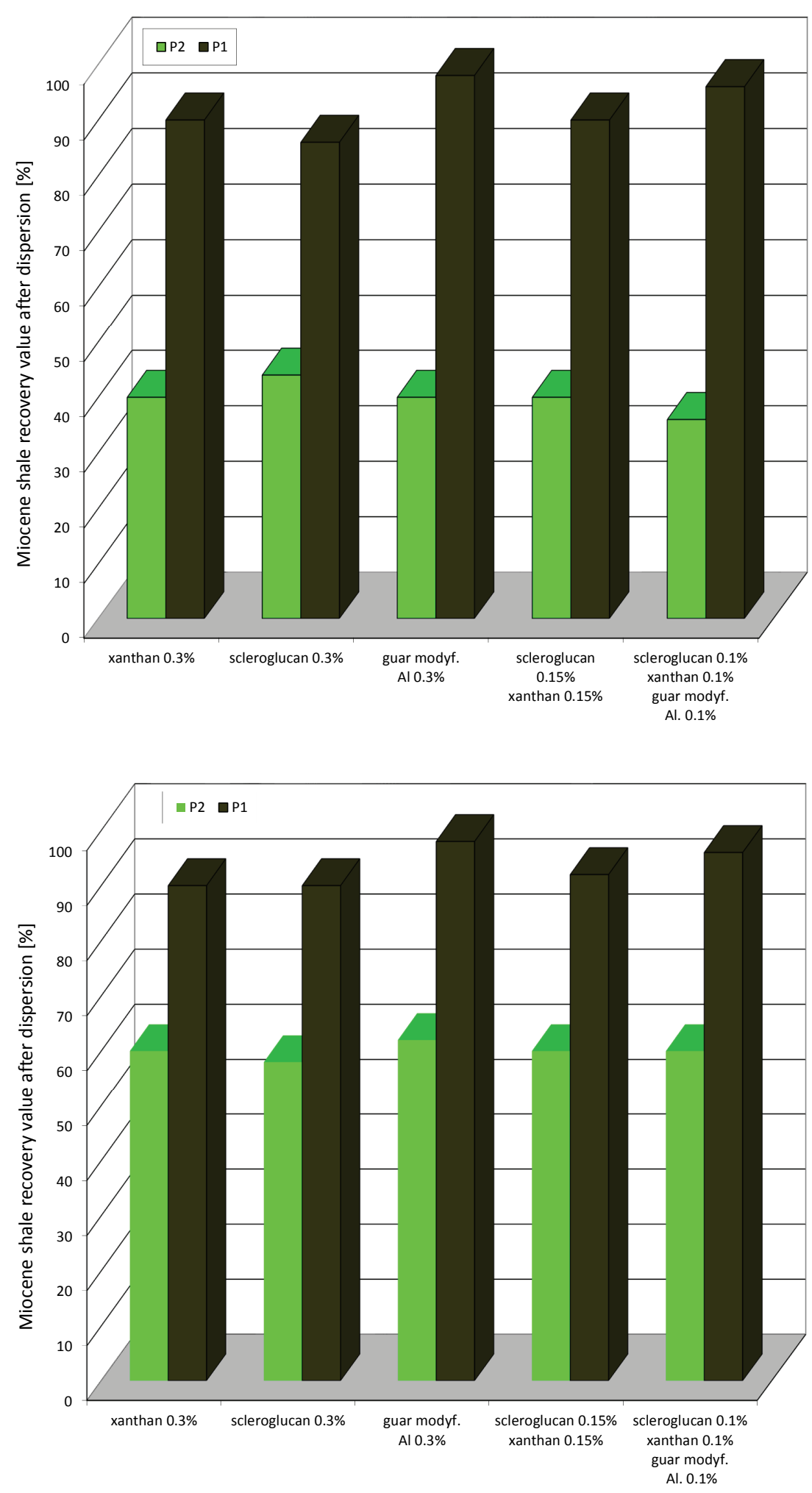

Fig. 1. The results of dispersion analysis on Miocene Shale in the environment of drilling muds containing various biopolymers; $P_{1}$ - shale recovery after dispersion in mud, $P_{2}-$ shale recovery after dispersion in water

Rys. 1. Wyniki analizy dyspersyjnej łupku mioceńskiego w środowisku płuczek zawierających różne biopolimery; $P_{1}$ - odzysk łupka po dyspergowaniu w płuczce, $P_{2}$ - odzysk łupka po dyspergowaniu w wodzie
Fig. 2. The results of Miocene Shale dispersion analysis on in the environment of drilling muds containing various biopolymers with addition $4 \%$ polyglycol; $P_{1}$ - shale recovery after dispersion in mud, $P_{2}-$ shale recovery after dispersion in water Rys. 2. Wyniki analizy dyspersyjnej łupku mioceńskiego w środowisku płuczek zawierających różne biopolimery z dodatkiem $4 \%$ poliglikolu; $P_{1}$ - odzysk łupka po dyspergowaniu w płuczce, $P_{2}$ - odzysk łupka po dyspergowaniu w wodzie

\section{Laboratory tests - the influence of temperature on the rheological parameters of the developed drilling muds}

Research on the change in rheological parameters of drilling muds under the influence of temperature carried out with the OFITE 77 viscometer (Fig. 3-22) confirmed the results obtained during heating in autoclaves. The rheological pa- rameters of the drilling muds with the addition of scleroglucan slightly decreased due to the temperature rise. Upon cooling, an increase in rheological parameters was observed in relation to the corresponding values when heated at given temperature.

The rheological parameters of the drilling mud with the addition of xanthan returned to the initial values. On the other hand the parameters of the drilling mud with the addition of scleroglucan were increased due to better hydration of the biopolymer. 


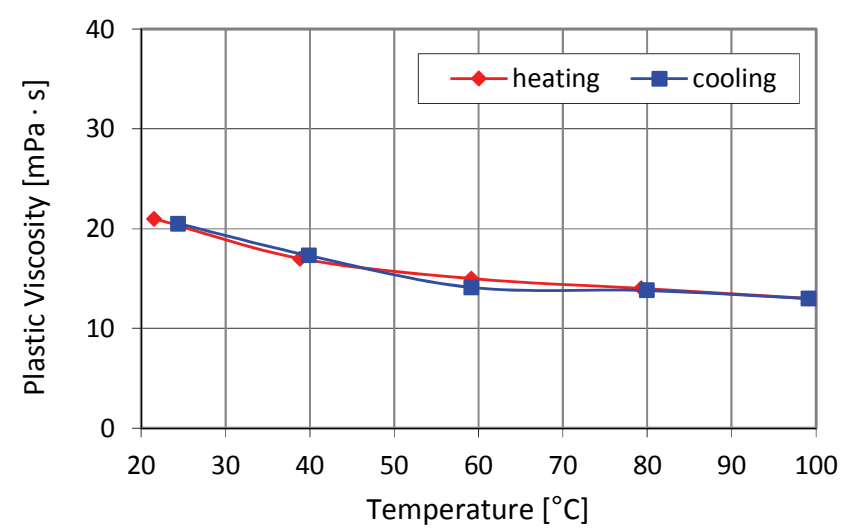

Fig. 3. Plastic Viscosity changes in the drilling mud containing $0.3 \%$ xanthan versus the temperature range $20-100^{\circ} \mathrm{C}$ plot

Rys. 3. Wykres zmian lepkości plastycznej płuczki zawierającej $0,3 \%$ ksantanu od temperatury w zakresie $20-100^{\circ} \mathrm{C}$

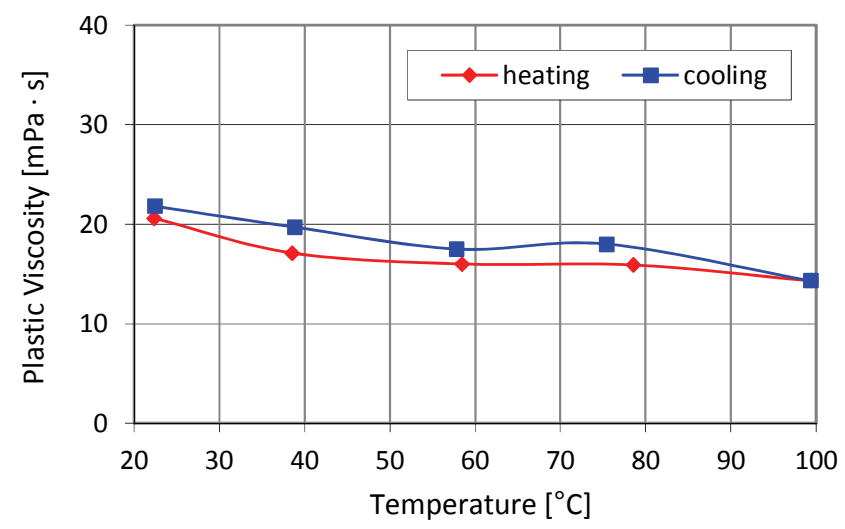

Fig. 5. Plastic Viscosity changes in the drilling mud containing $0.3 \%$ guar gum modified with aluminum ions versus the temperature range $20-100^{\circ} \mathrm{C}$ plot

Rys. 5. Wykres zmian lepkości plastycznej płuczki zawierającej $0,3 \%$ guaru modyfikowanego jonami glinu od temperatury w zakresie $20-100^{\circ} \mathrm{C}$

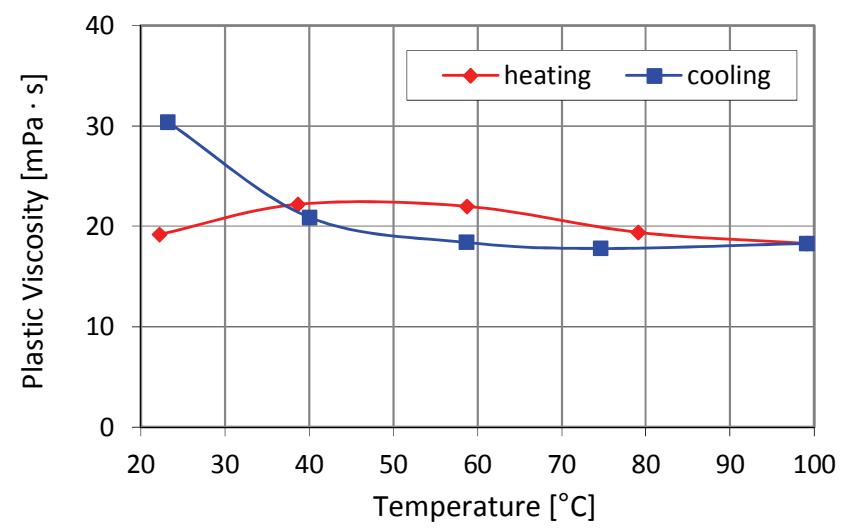

Fig. 7. Plastic Viscosity changes in the drilling mud containing $0.3 \%$ scleroglucan versus the temperature range $20-100^{\circ} \mathrm{C}$ plot

Rys. 7. Wykres zmian lepkości plastycznej płuczki zawierającej $0,3 \%$ skleroglukanu od temperatury w zakresie $20-100^{\circ} \mathrm{C}$

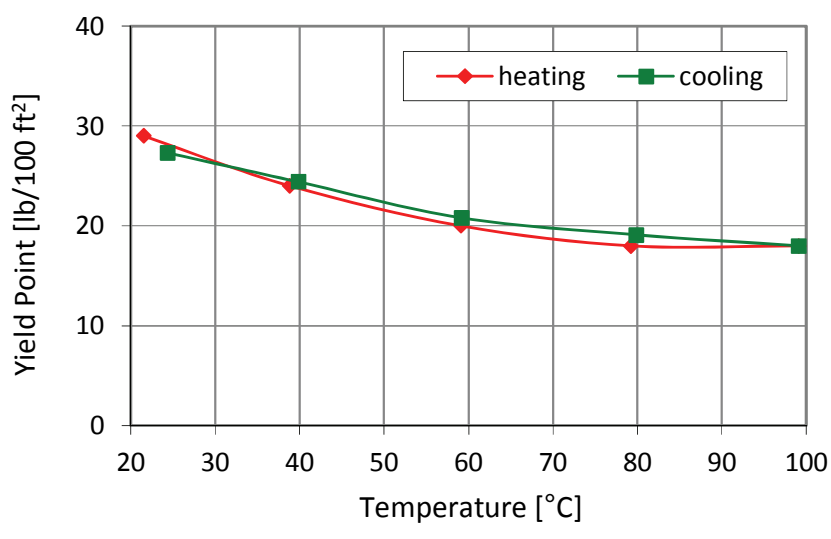

Fig. 4. Yield Point changes in the drilling mud containing $0.3 \%$ xanthan versus the temperature range $20-100^{\circ} \mathrm{C}$ plot

Rys. 4. Wykres zmian granicy płynięcia płuczki zawierającej $0,3 \%$ ksantanu od temperatury w zakresie $20-100^{\circ} \mathrm{C}$

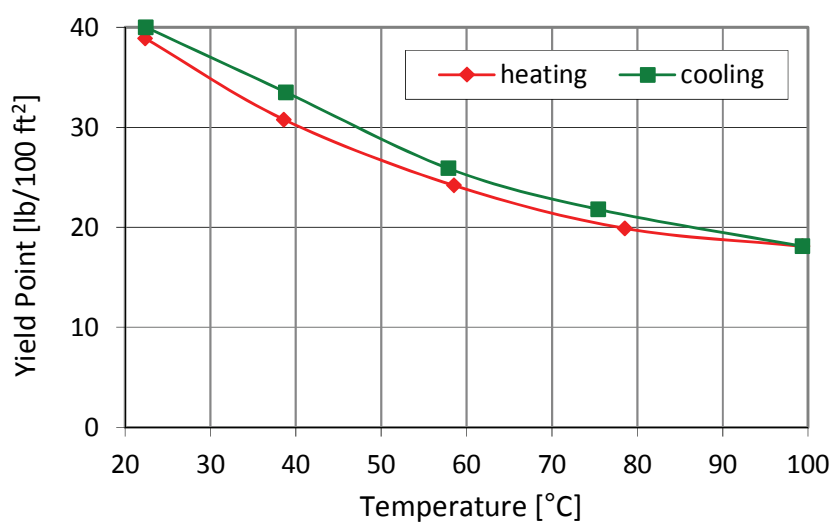

Fig. 6. Yield Point changes in the drilling mud containing $0.3 \%$ guar gum modified with aluminum ions verus the temperature range $20-100^{\circ} \mathrm{C}$ plot

Rys. 6. Wykres zmian granicy płynięcia płuczki zawierającej $0,3 \%$ guaru modyfikowanego jonami glinu od temperatury w zakresie $20-100^{\circ} \mathrm{C}$

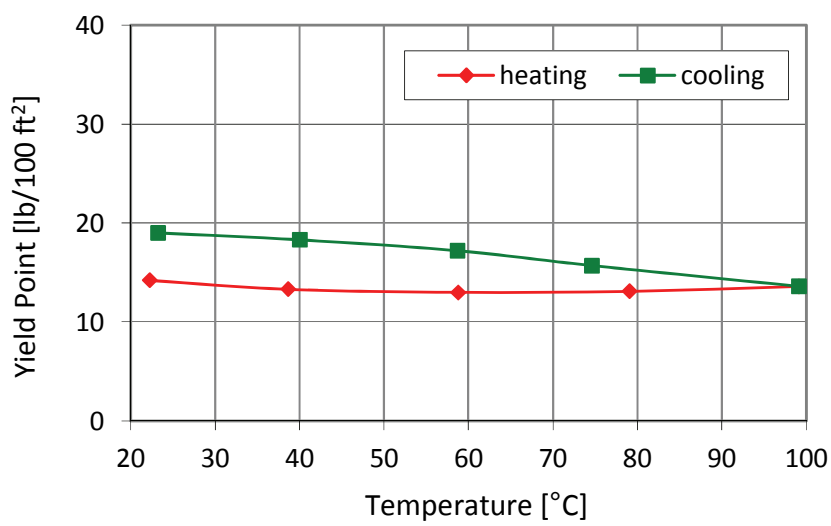

Fig. 8. Yield Point changes in the drilling mud containing $0.3 \%$ scleroglucan versus the temperature range of $20-100^{\circ} \mathrm{C}$ plot

Rys. 8. Wykres zmian granicy płynięcia płuczki zawierającej $0,3 \%$ skleroglukanu od temperatury w zakresie $20-100^{\circ} \mathrm{C}$ 


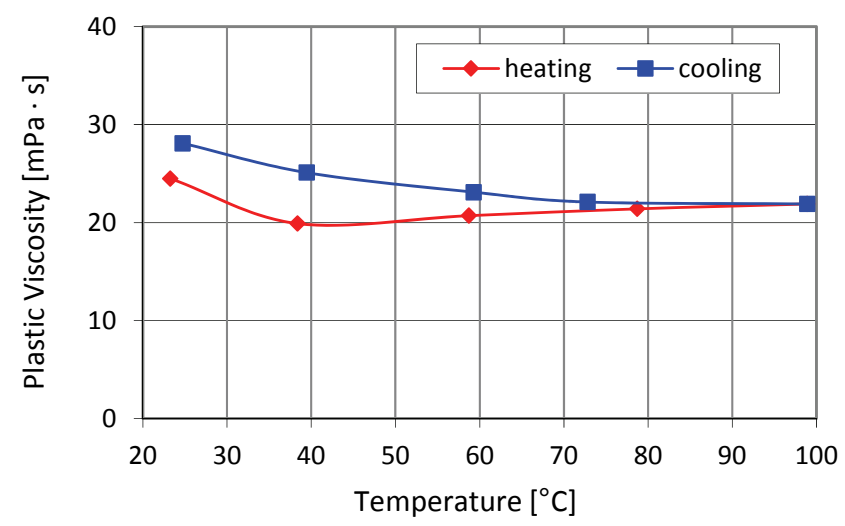

Fig. 9. Plastic Viscosity changes in the drilling mud containing $0.15 \%$ xanthan and $0.15 \%$ scleroglucan versus the temperature range $20-100^{\circ} \mathrm{C}$ plot

Rys. 9. Wykres zmian lepkosci plastycznej płuczki zawierającej $0,15 \%$ ksantanu i $0,15 \%$ skleroglukanu od temperatury w zakresie $20-100^{\circ} \mathrm{C}$

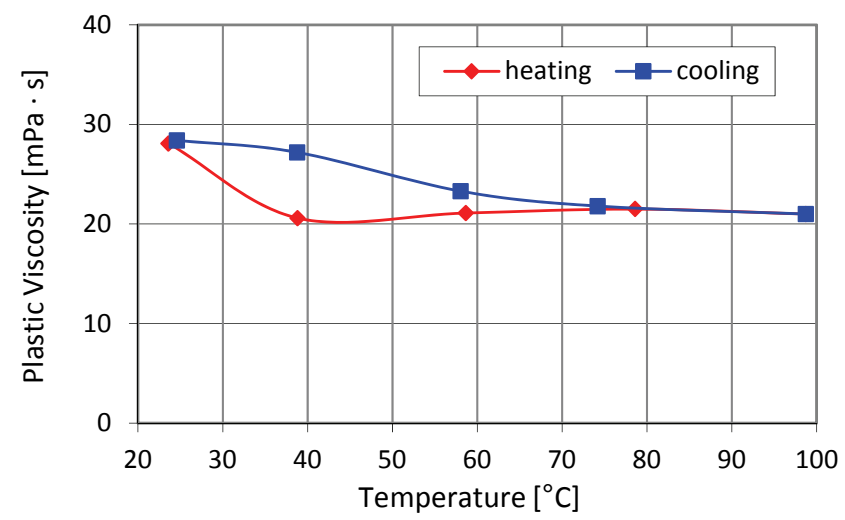

Fig. 11. Plastic Viscosity changes in the drilling mud containing $0.1 \%$ xanthan, 0,1 scleroglucan and 0.1 guar gum modified with aluminum ions versus the temperature range $20-100^{\circ} \mathrm{C}$ plot

Rys. 11. Wykres zmian lepkości plastycznej płuczki zawierającej 0,1\% ksantanu, 0,1 skleroglukanu i 0,1 guaru modyfikowanego jonami glinu od temperatury w zakresie $20-100^{\circ} \mathrm{C}$

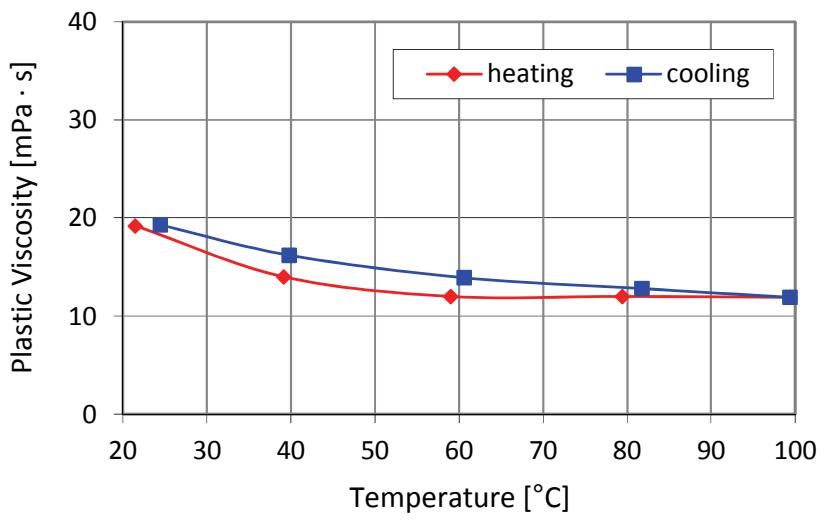

Fig. 13. Plastic Viscosity changes in the drilling mud containing $0.3 \%$ xanthan with addition $4 \%$ polyglycol versus the temperature range $20-100^{\circ} \mathrm{C}$ plot

Rys. 13. Wykres zmian lepkości plastycznej płuczki zawierającej $0,3 \%$ ksantanu z dodatkiem $4 \%$ poliglikolu od temperatury w zakresie $20-100^{\circ} \mathrm{C}$

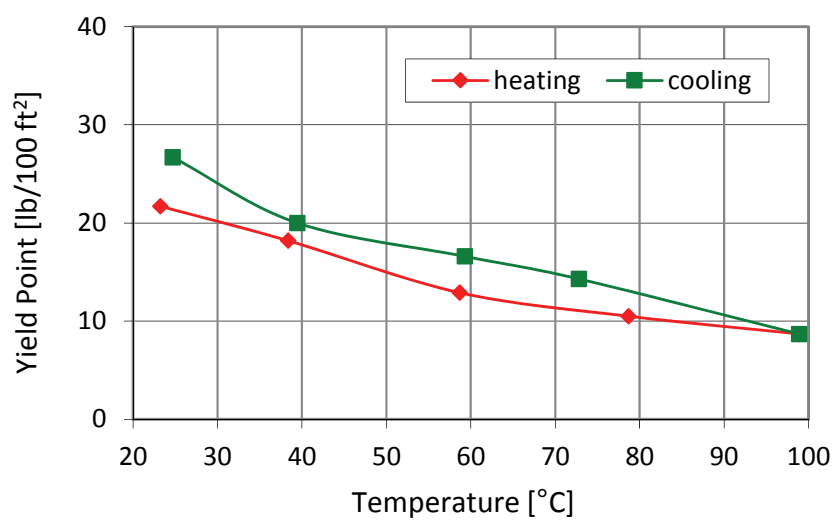

Fig. 10. Yield Point changes in the drilling mud containing $0.15 \%$ xanthan and $0.15 \%$ sclegorlucan versus the temperature range $20-100^{\circ} \mathrm{C}$ plot

Rys. 10. Wykres zmian granicy płynięcia płuczki zawierającej $0,15 \%$ ksantanu i $0,15 \%$ skleroglukanu od temperatury w zakresie $20-100^{\circ} \mathrm{C}$

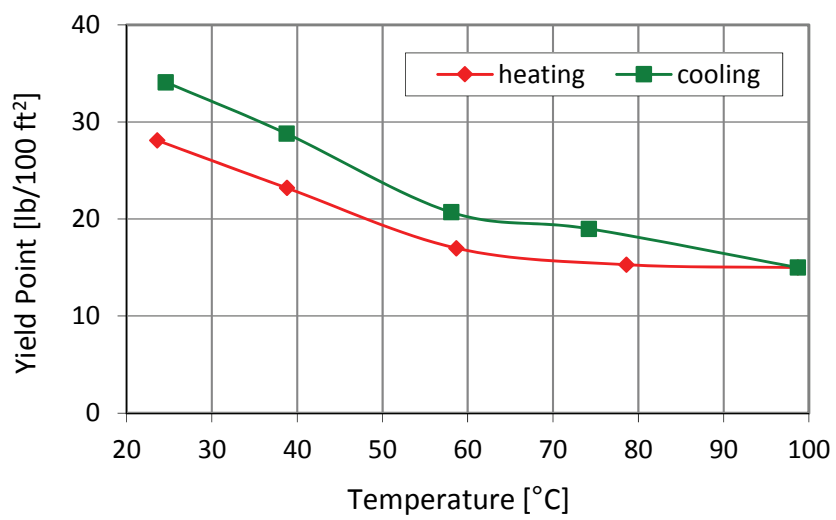

Fig. 12. Yield Point changes in the drilling mud containing $0.1 \%$ xanthan, 0.1 scleroglucan and $0,1 \%$ guar gum modified with aluminum ions versus the temperature range $20-100^{\circ} \mathrm{C}$ plot

Rys. 12. Wykres zmian granicy płynięcia płuczki zawierającej 0,1\% ksantanu, 0,1 skleroglukanu i 0,1 guaru modyfikowanego jonami glinu od temperatury w zakresie $20-100^{\circ} \mathrm{C}$

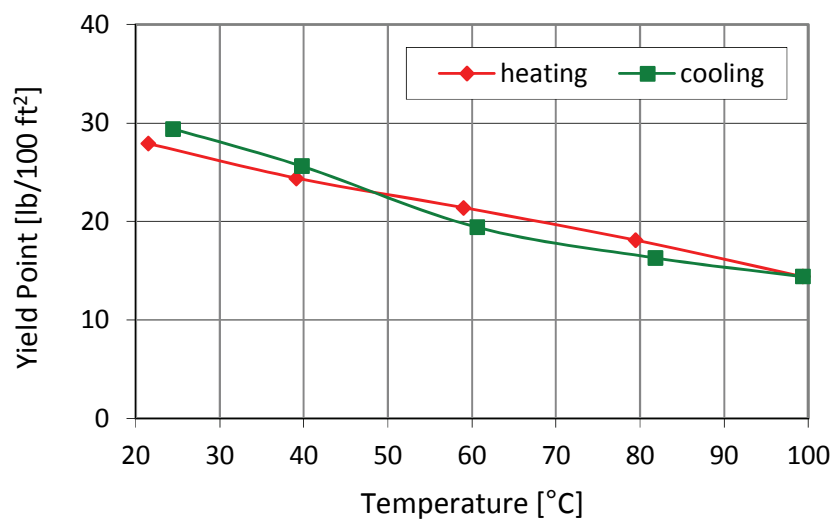

Fig. 14. Yield Point changes in the drilling mud containing $0.3 \%$ xanthan with addition $4 \%$ polyglycol versus the temperature range $20-100^{\circ} \mathrm{C}$ plot

Rys. 14. Wykres zmian granicy płynięcia płuczki zawierającej $0,3 \%$ ksantanu $\mathrm{z}$ dodatkiem $4 \%$ poliglikolu od temperatury $\mathrm{w}$ zakresie $20-100^{\circ} \mathrm{C}$ 


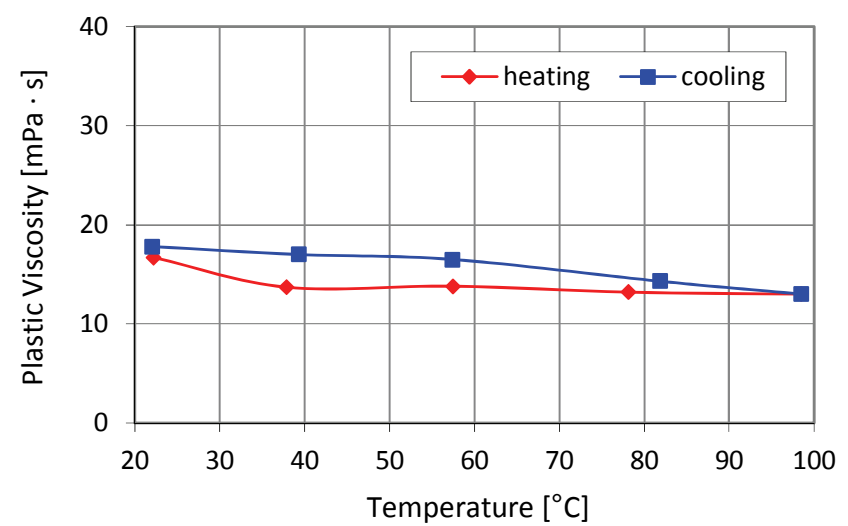

Fig. 15. Plastic Viscosity changes in the drilling mud containing $0.3 \%$ guar gum modified with aluminum ions with additional $4 \%$ polyglycol versus the temperature range $20-100^{\circ} \mathrm{C}$ plot

Rys. 15. Wykres zmian lepkości plastycznej płuczki zawierającej $0,3 \%$ guaru modyfikowanego jonami glinu z dodatkiem $4 \%$ poliglikolu od temperatury w zakresie $20-100^{\circ} \mathrm{C}$

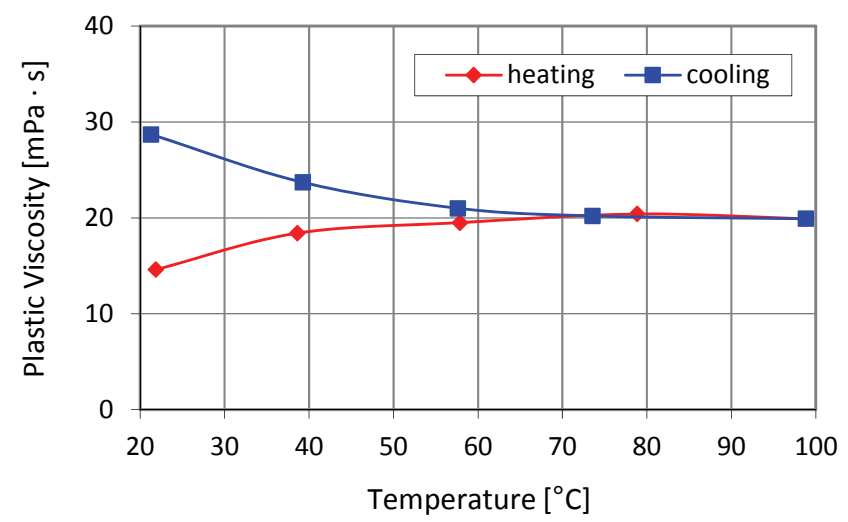

Fig. 17. Plastic Viscosity changes in the drilling mud containing $0.3 \%$ scleroglucan with additional $4 \%$ polyglycol versus the temperature range $20-100^{\circ} \mathrm{C}$ plot

Rys. 17. Wykres zmian lepkości plastycznej płuczki zawierającej $0,3 \%$ skleroglukanu $\mathrm{z}$ dodatkiem $4 \%$ poliglikolu od temperatury w zakresie $20-100^{\circ} \mathrm{C}$

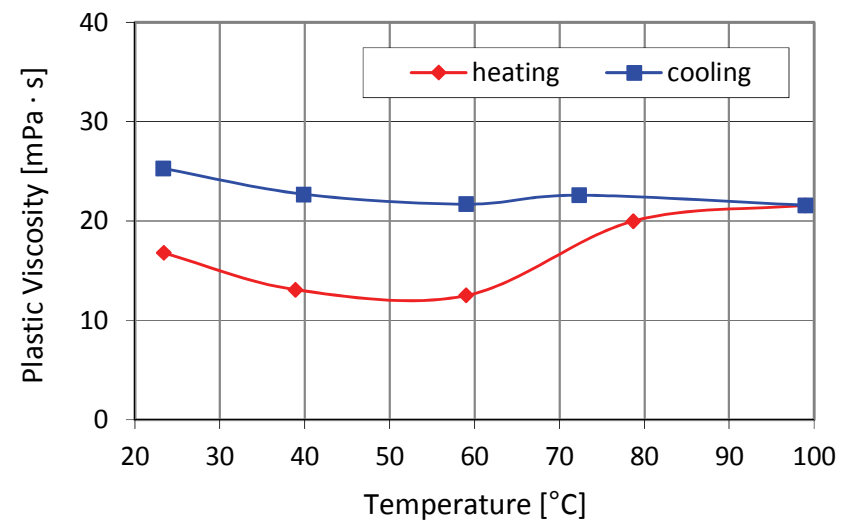

Fig. 19. Plastic Viscosity changes in the drilling mud containing $0.15 \%$ xanthan and 0,15 scleroglucan with additional $4 \%$ polyglycol versus the temperature range $20-100^{\circ} \mathrm{C}$ plot

Rys. 19. Wykres zmian lepkości plastycznej płuczki zawierającej $0,15 \%$ ksantanu i 0,15 skleroglukanu z dodatkiem $4 \%$ poliglikolu od temperatury w zakresie $20-100^{\circ} \mathrm{C}$

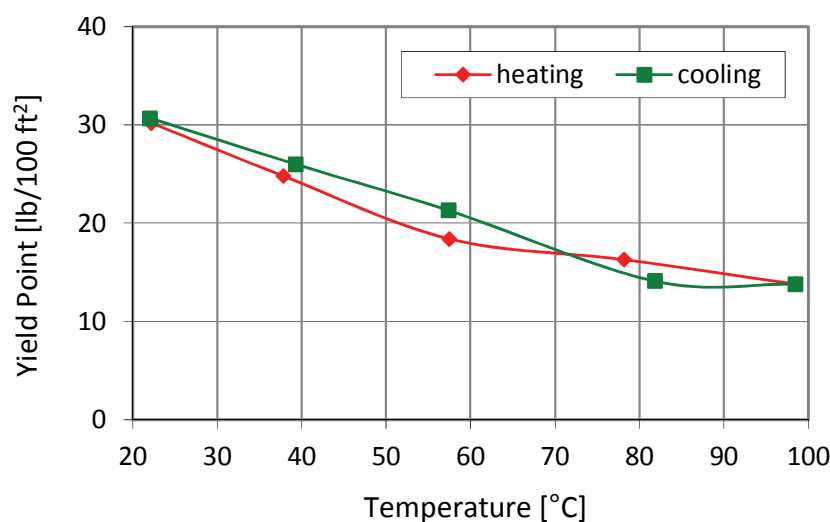

Fig. 16. Yield Point changes in the drilling mud containing $0.3 \%$ guar gum modified with aluminum ions with additional $4 \%$ polyglycol versus the temperature range $20-100^{\circ} \mathrm{C}$ plot

Rys. 16. Wykres zmian granicy płynięcia płuczki zawierającej $0,3 \%$ guaru modyfikowanego jonami glinu $\mathrm{z}$ dodatkiem $4 \%$ poliglikolu od temperatury w zakresie $20-100^{\circ} \mathrm{C}$

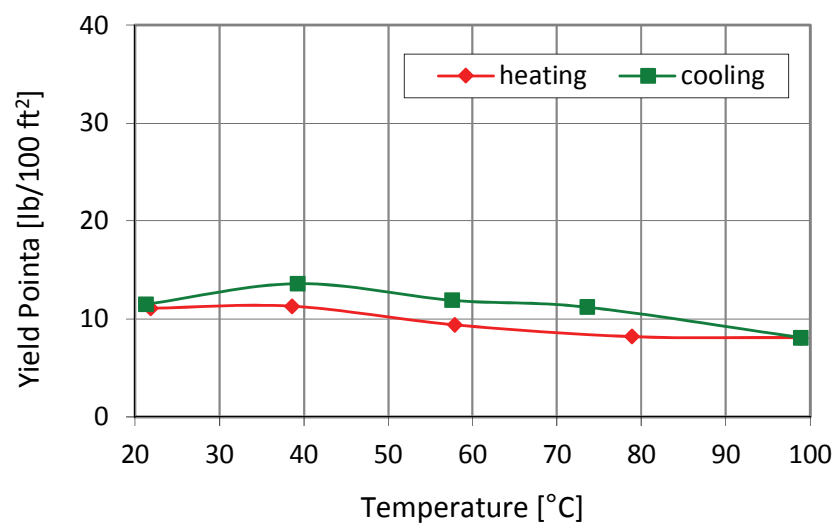

Fig. 18. Yield Point changes in the drilling mud containing $0.3 \%$ scleroglucan with additional $4 \%$ polyglycol versus the temperature range $20-100^{\circ} \mathrm{C}$ plot

Rys. 18. Wykres zmian granicy płynięcia płuczki zawierającej $0,3 \%$ skleroglukanu z dodatkiem $4 \%$ poliglikolu od temperatury w zakresie $20-100^{\circ} \mathrm{C}$

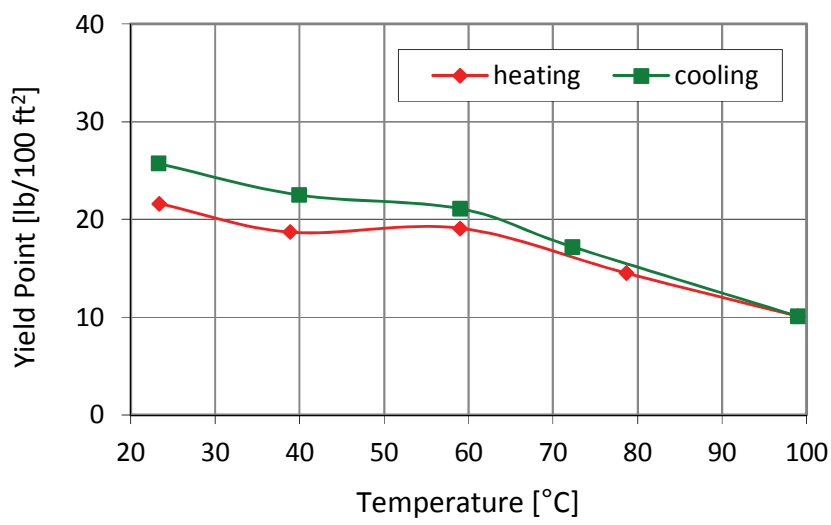

Fig. 20. Yield Point changes in the drilling mud containing $0.15 \%$ xanthan and 0.15 scleroglucan with additional $4 \%$ polyglycol versus the temperature range $20-100^{\circ} \mathrm{C}$ plot

Rys. 20. Wykres zmian granicy płynięcia płuczki zawierającej $0,15 \%$ ksantanu i 0,15 skleroglukanu z dodatkiem $4 \%$ poliglikolu od temperatury w zakresie $20-100^{\circ} \mathrm{C}$ 


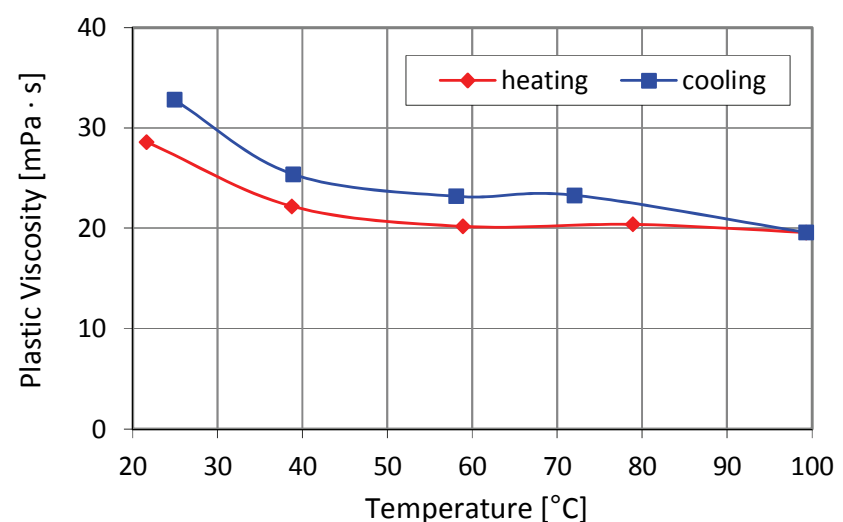

Fig. 21. Plastic Viscosity changes in the drilling mud containing $0.1 \%$ xanthan, 0.1 scleroglucan and 0,1 guar gum modified with aluminum ions and additional polyglycol versus the temperature range $20-100^{\circ} \mathrm{C}$ plot

Rys. 21. Wykres zmian lepkości plastycznej płuczki zawierającej $0,1 \%$ ksantanu, 0,1 skleroglukanu i 0,1 guaru modyfikowanego jonami glinu $\mathrm{z}$ dodatkiem poliglikolu od temperatury w zakresie $20-100^{\circ} \mathrm{C}$

Analysis of changes in rheological parameters, i.e. Plastic Viscosity and Yield Point in the range of $20-100^{\circ} \mathrm{C}$, indicates that the best stability of both parameters is provided by drilling muds containing additives of xanthan, scleroglucan and guar gum modified with aluminum ions both without the addition of polyglycol (Fig. 11, 12) and with polyglycol (Fig. 21, 22). The Plastic Viscosity of these drilling muds is practically constant within the temperature range of $40-100^{\circ} \mathrm{C}$, also the Yield Point remains stable in the range of $60-100^{\circ} \mathrm{C}$. The rheological parameters of the drilling mud with the addition of $0.3 \%$ scleroglucan also show stability in the considered temperature range (Fig. 7, 8, 17, 18). Changes in the Yield Point variance are insignificant, Plastic Viscosity changes considerably only in the range of $20-40^{\circ} \mathrm{C}$, but this applies to the PV comparison for the heating and cooling cycle. In drilling muds containing xanthan (Fig. 3, 4, 13, 14) and drilling muds containing guar gum modified with aluminum ions (Fig. 5, 6, 15, 16) Plastic Viscosity changes slightly, but a large change in Yield Point is observed, from a dozen or so to over $20 \mathrm{lb} / 100 \mathrm{ft}^{2}$. In the drilling muds containing $0.15 \%$ xanthan and scleroglucan (Fig. 9, $10,19,20)$ a significant change in the Yield Point is observed, but the Plastic Viscosity remains stable.

\section{Summary}

In the drilling muds compositions, the xanthan biopolymer (XCD) can be replaced by a different type of biopolymer such as scleroglucan or guar gum modified with aluminum ions without adversely affecting the properties of the drilling muds. We can control the rheological parameters of the drilling muds by com-

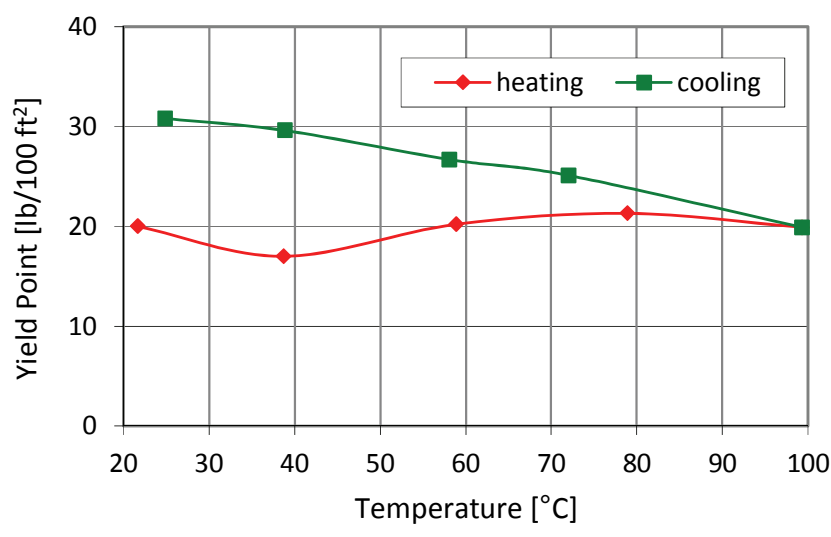

Fig. 22. Yield Point changes in the drilling mud containing $0.1 \%$ xanthan, 0.1 scleroglucan and 0.1 guar gum modified with aluminum ions and additional polyglycol versus the temperature range $20-100^{\circ} \mathrm{C}$ plot

Rys. 22. Wykres zmian granicy płynięcia płuczki zawierającej $0,1 \%$ ksantanu, 0,1 skleroglukanu i 0,1 guaru modyfikowanego jonami glinu $\mathrm{z}$ dodatkiem poliglikolu od temperatury $\mathrm{w}$ zakresie $20-100^{\circ} \mathrm{C}$

bining several biopolymers of various properties in the drilling muds composition. By changing the type and concentration of individual polymers, we can design rheological parameters of drilling muds in the selected temperature range. Scleroglucan shows some low values of rheological parameters after initial hydration in water but proper values of these parameters are obtained after heating at high temperature. Drilling muds prepared on the base of starch colloids with the addition of biopolymers do not protect the shales against hydration sufficiently. The recovery values of the shale formations after the dispersion test in water were about $40 \%$ for the tested drilling muds. The addition of polyglycol resulted in an increase in shale recovery after dispersing in water to $60 \%$. It also lowered level of water filtration in all the drilling muds tested by an average of $30 \%$. This addition has also positively influenced the stability of rheological parameters at high temperatures. Drilling muds containing guar gum modified with aluminum ions or scleroglucan show lower filtration levels than other drilling muds considered.

As part of the research, the compositions of drilling muds characterized by special stability of rheological parameters at high temperature were proposed. The most beneficial properties of the drilling mud were obtained using a combination of xanthan, scleroglucan and guar gum modified aluminum ions in its composition. The obtained results indicate the further direction of research on various biopolymers and synthetic polymers usage to design rheological parameters of the drilling mud in the selected temperature range.

This papier was written on the basis of the statutory work entitled: Analysis on the possible use of modified natural polymers 
to develop drilling muds - the work of the Oil and Gas Institute - National Research Institute was commissioned by the Ministry of Science and Higher Education; order number: 0060/KW/2017, archive number: DK-4100-47/2017.

\section{References}

Aston M.S., Elliott G.P., 1994. Water based glycol drilling muds: shale inhibition mechanism. Society of Petroleum Engineers. DOI: $10.2118 / 28818-M S$.

Behr A., Rafiee M., 2017. Prediction of Polymer Injectivity in Damaged Wellbore by Using Rheological Dependent Skin. Society of Petroleum Engineers. DOI: 10.2118/186054-MS.

Bielewicz D., Bortel E., 2000. Polymers in drilling fluids technology. Uczelniane Wydawnictwa Naukowo-Dydaktyczne, Akademia Górniczo-Hutnicza, Kraków.

Brattekås B., Steinsbø M., Graue A., Fernø M.A., Espedal H., Seright R.S., 2016. New Insight Into Wormhole Formation in Polymer Gel During Water Chase Floods With Positron Emission Tomography. Society of Petroleum Engineers. DOI: 10.2118/180051-MS.

Cannella W.J., Huh C., Seright R.S., 1988. Prediction of Xanthan Rheology in Porous Media. Society of Petroleum Engineers. DOI: 10.2118/18089-MS.

Clark P.E., Balakrishnan M., Sundram L., 1993. Crosslinking of Hydroxypropyl Guar With Metal Ions. Society of Petroleum Engineers. DOI: 10.2118/25208-MS.

Dawson J.C., 1991. A Thermodynamic Study of Borate Complexation With Guar and Guar Derivatives. Society of Petroleum Engineers. DOI: 10.2118/22837-MS.

de Kruijf A.S., Roodhart L.P., Davies D.R., 1993. Relation Between Chemistry and Flow Mechanics of Borate-Crosslinked Fracturing Fluids. Society of Petroleum Engineers. DOI: 10.2118/25206-PA.

Delshad M., Kim D.H., Magbagbeola O.A., Huh C., Pope G.A., Tarahhom F., 2008. Mechanistic Interpretation and Utilization of Viscoelastic Behavior of Polymer Solutions for Improved Polymer-Flood Efficiency. Society of Petroleum Engineers. DOI: 10.2118/113620-MS.

Dupas A., Henaut I., Rousseau D., Poulain P., Tabary R., Argillier J.-F., Aubry T., 2013. Impact of Polymer Mechanical Degradation on Shear and Extensional Viscosities: Toward Better Injectivity Forecasts in Polymer Flooding Operations. Society of Petroleum Engineers. DOI: 10.2118/164083-MS.

El-Karsani K.S.M, Al-Muntasheri G.A., Hussein I.A., 2014a. Polymer systems for water shutoff and profile modification: a review over the last decade. Society of Petroleum Engineers. DOI: 10.2118/163100-PA.

El-Karsani K.S.M., Al-Muntasheri G.A., Sultan A.S., Hussein I.A., 2014b. Gelation of a water-shutoff gel at high pressure and high temperature: rheological investigation. Society of Petroleum Engineers. DOI: 10.2118/173185-PA.

Eriksen O.I., Daasvatn K., Vigerust B., Braen T., Olafsen K., MoradiAraghi A., Hamouda A.A., 1997. Gel formation and thermal stability of gels made from novel water-soluble polymers for enhanced oil recovery applications. Society of Petroleum Engineers. DOI: 10.2118/37247-MS.

Fjelde I., Stavland A., 1995. Scleroglucan Gels for Profile Modifications in High-Temperature Reservoirs. Society of Petroleum Engineers. DOI: 10.2118/25223-PA.

Fortenberry R., Hernandez R., Li Z., Delshad M., 2017. Customizing Polymer Rheology for Application in Commercial Scale Polymer Floods. Society of Petroleum Engineers. DOI: 10.2118/187303-MS.
Gaillard N., Giovannetti B., Leblanc T., Thomas A., Braun O., Favero C., 2015. Selection of Customized Polymers to Enhance Oil Recovery from High Temperature Reservoirs. Society of Petroleum Engineers. DOI: 10.2118/177073-MS.

Gallino G., Guarneri A., Poli G., Xiao L., 1996. Scleroglucan Biopolymer Enhances WBM Performances. Society of Petroleum Engineers. DOI: 10.2118/36426-MS.

Harris P.C., 1993. Chemistry and Rheology of Borate-Crosslinked Fluids at temperatures to $300^{\circ}$ F. Society of Petroleum Engineers. DOI: $10.2118 / 24339-P A$.

Himes R.E., Ali S.A., Hardy M.A., Holtmyer M.D., Weaver J.D. 1994. Reversible, Crosslinkable Polymer for Fluid-Loss Control. Society of Petroleum Engineers. DOI: 10.2118/27373-MS.

Howard S., Kaminski L., Downs J., 2015. Xanthan Stability in Formate Brines - Formulating Non-damaging Fluids for High Temperature Applications. Society of Petroleum Engineers. DOI: 10.2118/174228-MS.

Jouenne S., Chakibi H., Levitt D., 2015. Polymer Stability Following Successive Mechanical Degradation events. Society of Petroleum Engineers. DOI: 10.2118/186103-PA.

Kalpakci B., Magri N.F., Padolewski J.P., 1990. Thermal Stability of Scleroglucan at Realistic Reservoir Conditions. Society of Petroleum Engineers. DOI: 10.2118/20237-MS.

Kramer J., Prud'homme R.K., Norman L.R., Sandy J.M., 1987. Charakteristics of Metal-Polymer Interactions in Fracturing Fluids Systems. Society of Petroleum Engineers. DOI: 10.2118/16914-MS.

Kulawardana E.U., Koh H., Kim D.H., Liyanage P.J., Upamali K., Huh C., Pope G.A., 1989. Rheology and Transport of Improved EOR Polymers under Harsh Reservoir Conditions. Society of Petroleum Engineers. DOI: SPE 10.2118/154294-MS.

Lange P., Keilhofer G., 2004. Industrial Biopolymers for Oilwell Drilling: Xanthan Gum, Scleroglucan and how both differ at elevated temperatures. Degussa Construction Polymers GmbH, Trostberg, Germany.

Leonhardt B., Ernst B., Reimann S., Steigerwald A., Lehr F., 2014. Field Testing the Polysaccharide Schizophyllan: Results of the First Year. Society of Petroleum Engineers. DOI: 10.2118/169032-MS.

Li Z., Delshad M., 2014. Development of an Analytical Injectivity Model for Non-Newtonian Polymer Solutions. Society of Petroleum Engineers. DOI: 10.2118/163672-PA.

Lomba R.F.T., 2002. Drill-In Fluids: Identydying Invasion Mechanisms. Society of Petroleum Engineers. DOI: 10.2118/73714-MS.

Luyster M.R., Tresco K., Dobson J., Ravitz R., Eyaa Allogo C.M., Sooi Lim K., 2016. An Assessment of an Uncomplicated Drill-in Fluid and Its Application to a Wide Range of Global Completions and Their Recent Successes. Society of Petroleum Engineers. DOI: 10.2118/179037-MS.

Navarrete R.C., Coffey M.D., 2000. New Bio-polymers for Drilling, Drill-In, Completions, Spacer and Coiled Tubing Applications. Society of Petroleum Engineers. 10.2118/64982-MS.

Navarrete R.C., Himes R.E., Seheult J.M., 2000. Applications of Xanthan Gum in Fluid-Loss Control and Related Formation Damage. Society of Petroleum Engineers. DOI: 10.2118/59535-MS.

Ogezi O., Strobel J., Egbuniwe D., Leonhardt B., 2014. Operational Aspects of a Biopolymer Flood in a Mature Oilfield. Society of Petroleum Engineers. DOI: 10.2118/169158-MS.

Queiroz J., dos Santos R.L., 2000. Evolution of a Damaged Zone Caused by Water-Based Polymeric Drill-In Fluid. Society of Petroleum Engineers. DOI: 10.2118/58742-MS.

Rae P., di Lullo G., 1996. Fracturing Fluids and Breaker Systems 
- A Review of the State-of-the-Art. Society of Petroleum Engineers. DOI: 10.2118/37359-MS.

Rivenq R.C., Nolk C., 1992. Improwed Scleroglucan for Polymer Flooding Under Harsh Reservoir Conditions. Society of Petroleum Engineers. DOI: 10.2118/19635-PA.

Seright R.S., Seheult J.M., Talashek T., 2009. Injectivity Characteristics of EOR Polymers. Society of Petroleum Engineers. DOI: 10.2118/115142-PA.

Shaari N.E., Kedzierski M., Gorham T.L., 2005. Quantyfying Guar Polymer Recovery Post Hydraulic Fracturing to Determine the Degree of Fracture Cleanup: A Field Study of the Point of Rocks Formation. Society of Petroleum Engineers. DOI: 10.2118/93912-MS.

Steliga T., Uliasz M., 2012. Wybrane zagadnienia środowiskowe podczas poszukiwania, udostępniania i eksploatacji gazu ziemnego z formacji łupkowych. Nafta-Gaz, 5: 273-283.

Suresh Kumar M., Pandey A., Jha M.K.: 2012. Polymer Injectivity Test in Mangala Field - A Significant Step towards Field Wide Implementation. Society of Petroleum Engineers. DOI: 10.2118/155162-MS.

Sydansk R.D., Southwell G.P., 2000. More than 12 years of experience with a successful conformance-control polymer geltechnology. Society of Petroleum Engineers. DOI: 10.2118/66558-PA.

Taugbol K., Svanes G., Svanes K., Omland T.H., Alteraas E., Mathisen A.M., 2005. Investigation of Flow-Back Properties of Various Drilling and Completion Fluids through Production Screens. Society of Petroleum Engineers. DOI: 10.2118/94558-MS. van Oort E., Bland R.G., Howard S.K., Wiersma R.J., Roberson L., 1997. Improving HPHT Stability of Water Based Drilling Fliuds. Society of Petroleum Engineers. DOI: 10.2118/37605-MS.
Wreath D., Pope G.A., Sepehrnoori, K., 1990. Dependence of Polymer Apparent Viscosity on the Permeable Media and Flow Conditions. In Situ, 14(3): 263-284.

Yerramilli S.S., Zitha P.L.J., Yerramilli R.C., 2013. Novel Insight into Polymer Injectivity for Polymer Flooding. Society of Petroleum Engineers. DOI: 10.2118/165195-MS

Yuan C., Delshad M., Wheeler M. F., 2010. Parallel Simulations of Commercial-Scale Polymer Floods. Society of Petroleum Engineers. DOI: 10.2118/132441-MS.

Zhu D., Jirui Hou, Qi Wei, Yuguang Chen, Kewen Peng, 2017. Development of a High-Temperature Resistant Polymer Gel System for Conformance Control in Jidong Oilfield. Society of Petroleum Engineers. DOI: 10.2118/186235-PA.

Zima G., 2001. Chemical modifications of natural polymers used in drilling jobs. Nafta-Gaz, 6: 321-325.

Zima G., Błaż S. 2004. Influence of drilling muds containing modified guar resins on rock properties in the light of laboratory experiments. XV Międzynarodowa Konferencja Naukowo-Techniczna, WWNiG AGH, Krynica, 16-18.06.2004.

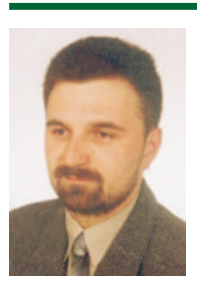
Grzegorz ZIMA Ph.D. Eng.

Assistant Professor Department of Drilling Technology Oil and Gas Institute - National Research Institute 25 A Lubicz St.

31-503 Krakow

E-mail: grzegorz.zima@inig.pl

\section{OFERTA BADAWCZA ZAKŁADU BADANIA ZKÓŻ ROPY I GAZU}

- pobór wgtębnych i powierzchniowych próbek płynów ztożowych;

- kompleksowe badania i analizy zmian fazowych próbek płynów ztożowych na zestawie aparatów PVT firmy Chandler i Ruska;

- modelowanie procesu wypierania ropy gazem na fizycznym modelu ztoża tzw. „cienka rurka";

- pomiar lepkości ropy wiskozymetrem kulkowym lub kapilarnym w warunkach PT;

- optymalizacja procesów powierzchniowej separacji ropy naftowej;

- laboratoryjne i symulacyjne badania warunków wytrącania się parafin, asfaltenów w ropie oraz tworzenia się hydratów w gazie;

- badanie skuteczności dziatania chemicznych środków zapobiegaiących tworzeniu się hydratów;

- laboratoryjne modelowanie procesów wypierania ropy gazem w warunkach zmieszania faz;

- badanie procesów sekwestracji $\mathrm{CO}_{2}$ w solankowych poziomach wodonośnych, nasyconych gazem ziemnym;

- badania na dtugich rdzeniach wiertniczych dla oceny efektywności metod zwiększenia stopnia odzysku ropy.

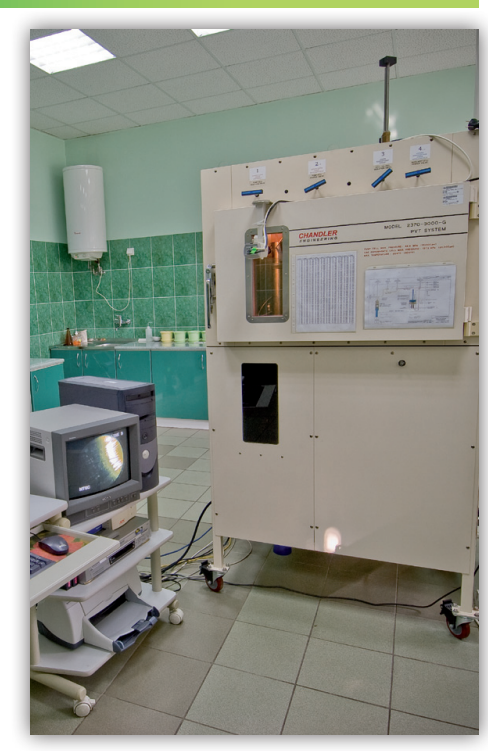

INSTYTUT NAFTY I GAZU - Państwowy Instytut Badawczy 